Cochrane Database of Systematic Reviews

\title{
Negative pressure wound therapy for treating leg ulcers (Review)
}

Dumville JC, Land L, Evans D, Peinemann F

Dumville JC, Land L, Evans D, Peinemann F.

Negative pressure wound therapy for treating leg ulcers.

Cochrane Database of Systematic Reviews 2015, Issue 7. Art. No.: CD011354.

DOI: 10.1002/14651858.CD011354.pub2.

www.cochranelibrary.com 
TABLE OF CONTENTS

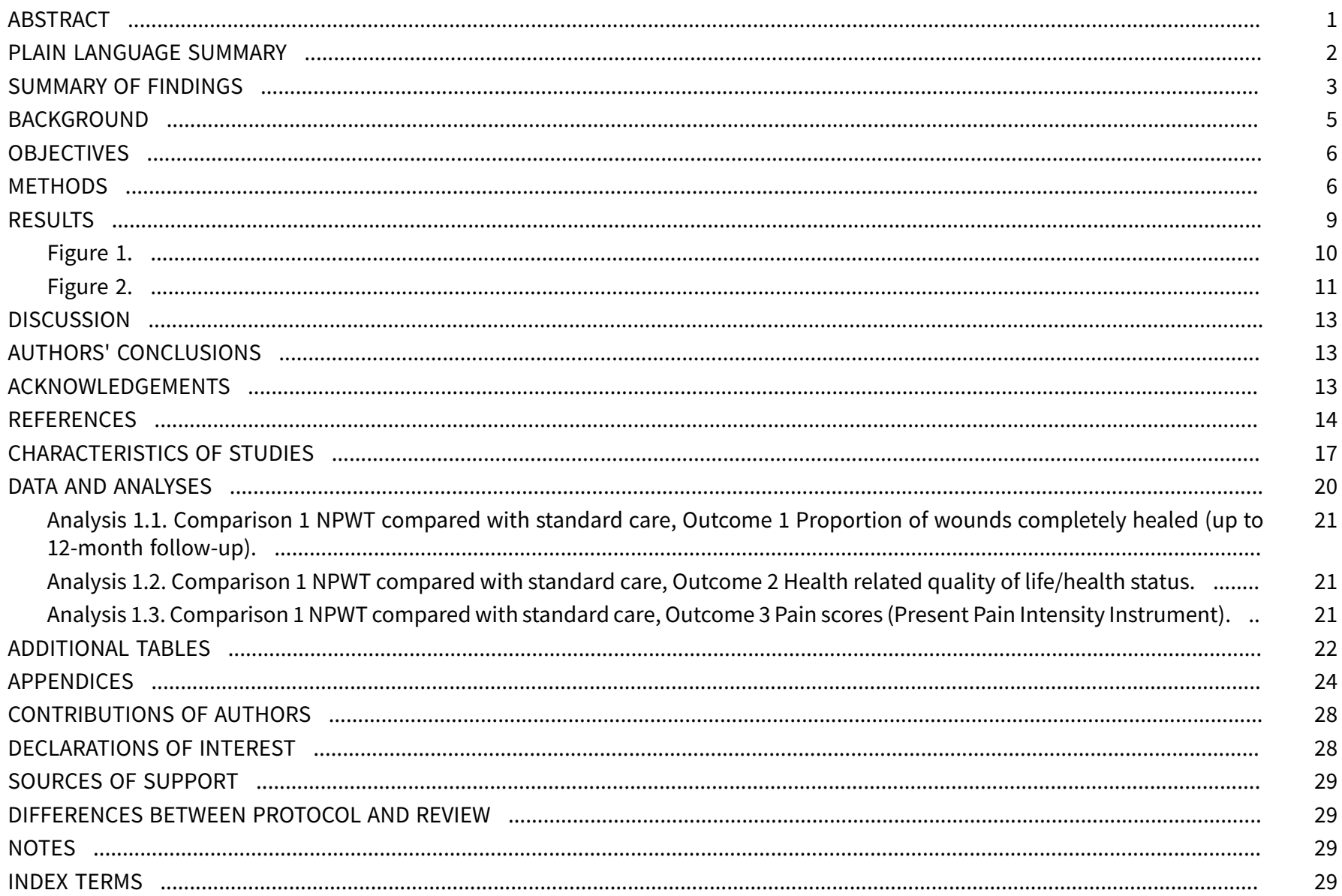


[Intervention Review]

\title{
Negative pressure wound therapy for treating leg ulcers
}

\author{
Jo C Dumville1, Lucy Land², Debra Evans³ ${ }^{3}$ Frank Peinemann4
}

1Division of Nursing, Midwifery \& Social Work, School of Health Sciences, Faculty of Biology, Medicine \& Health, University of Manchester, Manchester, UK. ${ }^{2}$ Centre for Health and Social Care Research, Birmingham City University, Birmingham, UK. ${ }^{3}$ Division of Community Health and Social Work, Birmingham City University, Birmingham, UK. ${ }^{4}$ Pediatric Oncology and Hematology, Children's Hospital, University of Cologne, Cologne, Germany

Contact: Jo C Dumville, Division of Nursing, Midwifery \& Social Work, School of Health Sciences, Faculty of Biology, Medicine \& Health, University of Manchester, Manchester, M13 9PL, UK. jo.dumville@manchester.ac.uk.

Editorial group: Cochrane Wounds Group.

Publication status and date: Edited (no change to conclusions), published in Issue 12, 2016.

Citation: Dumville JC, Land L, Evans D, Peinemann F. Negative pressure wound therapy for treating leg ulcers. Cochrane Database of Systematic Reviews 2015, Issue 7. Art. No.: CD011354. DOI: 10.1002/14651858.CD011354.pub2.

Copyright @ 2016 The Cochrane Collaboration. Published by John Wiley \& Sons, Ltd.

\section{A B S T R A C T}

\section{Background}

Leg ulcers are open skin wounds that occur between the ankle and the knee that can last weeks, months or even years and are a consequence of arterial or venous valvular insufficiency. Negative pressure wound therapy (NPWT) is a technology that is currently used widely in wound care and is promoted for use on wounds. NPWT involves the application of a wound dressing to the wound, to which a machine is attached. The machine applies a carefully controlled negative pressure (or vacuum), which sucks any wound and tissue fluid away from the treated area into a canister.

\section{Objectives}

To assess the effects of negative pressure wound therapy (NPWT) for treating leg ulcers in any care setting.

\section{Search methods}

For this review, in May 2015 we searched the following databases: the Cochrane Wounds Group Specialised Register (searched 21 May 2015); the Cochrane Central Register of Controlled Trials (CENTRAL; The Cochrane Library 2015, Issue 4); Ovid MEDLINE (1946 to 20 May 2015); Ovid MEDLINE (In-Process \& Other Non-Indexed Citations 20 May 2015); Ovid EMBASE (1974 to 20 May 2015); EBSCO CINAHL (1982 to 21 May 2015). There were no restrictions based on language or date of publication.

\section{Selection criteria}

Published or unpublished randomized controlled trials (RCTs) comparing the effects of NPWT with alternative treatments or different types of NPWT in the treatment of leg ulcers.

\section{Data collection and analysis}

Two review authors independently performed study selection, risk of bias assessment and data extraction.

\section{Main results}

We included one study, with 60 randomized participants, in the review. The study population had a range of ulcer types that were venous arteriolosclerotic and venous/arterial in origin. Study participants had recalcitrant ulcers that had not healed after treatment over a six-month period. Participants allocated to NPWT received continuous negative pressure until they achieved 100\% granulation (wound preparation stage). A punch skin-graft transplantation was conducted and the wound then exposed to further NPWT for four days followed by standard care. Participants allocated to the control arm received standard care with dressings and compression until $100 \%$ granulation 
was achieved. These participants also received a punch skin-graft transplant and then further treatment with standard care. All participants were treated as in-patients until healing occurred.

There was low quality evidence of a difference in time to healing that favoured the NPWT group: the study reported an adjusted hazard ratio of 3.2, with $95 \%$ confidence intervals $(\mathrm{Cl}) 1.7$ to 6.2 . The follow-up period of the study was a minimum of 12 months. There was no evidence of a difference in the total number of ulcers healed (29/30 in each group) over the follow-up period; this finding was also low quality evidence.

There was low quality evidence of a difference in time to wound preparation for surgery that favoured NPWT (hazard ratio $2.4,95 \%$ Cl 1.2 to 4.7).

Limited data on adverse events were collected: these provided low quality evidence of no difference in pain scores and Euroqol (EQ-5D) scores at eight weeks after surgery.

\section{Authors' conclusions}

There is limited rigorous RCT evidence available concerning the clinical effectiveness of NPWT in the treatment of leg ulcers. There is some evidence that the treatment may reduce time to healing as part of a treatment that includes a punch skin graft transplant, however, the applicability of this finding may be limited by the very specific context in which NPWT was evaluated. There is no RCT evidence on the effectiveness of NPWT as a primary treatment for leg ulcers.

\section{PLAIN LANGUAGE SUMMARY}

\section{Negative pressure wound therapy for treating leg ulcers}

\section{Background}

Leg ulcers are wounds that occur between the ankle and the knee as a result of poor blood flow in the legs. These wounds are relatively common often affecting older people. There are several different treatments for these ulcers and the underlying problems that cause them. Negative pressure wound therapy (NPWT) is a treatment currently beng used for wounds including leg ulcers. NPWT involves the application to the wound of a dressing to which a machine is attached. The machine then applies a carefully controlled negative pressure (or vacuum), and sucks any wound and tissue fluid away from the treated area into a canister.

\section{What we found}

After extensive searching up to May 2015 to find all relevant medical studies that might provide evidence about whether NPWT is an effective treatment for leg ulcers, we found only one randomized controlled trial (RCT) that was eligible for this review. (RCTs provide more robust results than most other trial types.) The study was small with 60 participants who had hard-to-heal ulcers. The average age of these participants was 73 years, and $77 \%$ of them were women. The study was funded by the manufacturer of the NPWT machine. The study explored the use of NPWT in preparing leg ulcers for a skin graft. In the study, the ulcers were treated with NPWT or with normal (standard) care until the wounds were considered ready to have a skin graft applied. The study's results are not relevant for leg ulcers that are not being prepared for skin grafts. Participants remained in hospitals during treatment and until their wounds healed.

There was low evidence from this study that ulcers treated with NPWT healed more quickly than those treated with standard care (dressings and compression). There was also evidence that ulcers treated with NPWT became ready for skin grafting more quickly than those treated with standard care. There were very limited results for other outcomes such as adverse events (harms) and it was not clear how information about adverse effects was collected. Twelve ulcers recurred (broke out again) in the NPWT group and 10 recurred in the standard care group.

The evidence for the effectiveness of NPWT in treating leg ulcers is very limited, and at present consists of only one study with 60 participants. This study provided evidence that NPWT may reduce time to healing as part of a treatment that includes a skin graft. At present, no RCTs have investigated the effectiveness of NPWT as a main treatment for leg ulcers.

This plain language summary is up-to-date as of May 2015. 
SUMMARY OF FINDINGS

Summary of findings for the main comparison. Negative pressure wound therapy compared to standard care for people with leg ulcers undergoing a punch skin-graft

Negative pressure wound therapy compared to standard care for people with leg ulcers undergoing a punch skin-graft

Patient or population: people with leg ulcers undergoing a punch skin-graft

Settings: in-patient

Intervention: negative pressure wound therapy

Comparison: standard care

\begin{tabular}{|c|c|c|c|c|c|c|}
\hline \multirow[t]{3}{*}{ Outcomes } & \multicolumn{2}{|c|}{ Illustrative comparative risks* $(95 \% \mathrm{CI})$} & \multirow{3}{*}{$\begin{array}{l}\text { Relative effect } \\
(95 \% \mathrm{CI})\end{array}$} & \multirow{3}{*}{$\begin{array}{l}\text { No of partici- } \\
\text { pants } \\
\text { (studies) }\end{array}$} & \multirow{3}{*}{$\begin{array}{l}\text { Quality of the } \\
\text { evidence } \\
\text { (GRADE) }\end{array}$} & \multirow[t]{3}{*}{ Comments } \\
\hline & Assumed risk & Corresponding risk & & & & \\
\hline & Standard care & $\begin{array}{l}\text { Negative pressure wound } \\
\text { therapy }\end{array}$ & & & & \\
\hline $\begin{array}{l}\text { Time to heal- } \\
\text { ing }\end{array}$ & \multicolumn{2}{|c|}{ Study population } & $\begin{array}{l}\text { HR } 3.2 \\
95 \% \mathrm{Cl}(1.7 \text { to } \\
6.2)\end{array}$ & $\begin{array}{l}60 \\
\text { (1 study) }\end{array}$ & $\begin{array}{l}\oplus \oplus \odot \odot \\
\text { low } 1,2,3\end{array}$ & $\begin{array}{l}\text { The intervention group had a high haz- } \\
\text { ard of healing - thus a shorter time to } \\
\text { healing compared with the standard } \\
\text { care group. }\end{array}$ \\
\hline \multirow{2}{*}{$\begin{array}{l}\text { Proportion of } \\
\text { wounds healed }\end{array}$} & \multicolumn{2}{|c|}{ Study population } & \multirow{2}{*}{$\begin{array}{l}\text { RR } 1.00 \\
95 \% \mathrm{Cl}(0.91 \text { to } \\
1.10)\end{array}$} & \multirow{2}{*}{$\begin{array}{l}60 \\
\text { (1 study) }\end{array}$} & \multirow{2}{*}{$\begin{array}{l}\oplus \oplus \odot \ominus \\
\text { low } 3,4\end{array}$} & \\
\hline & 967 per 1000 & $\begin{array}{l}967 \text { per } 1000 \\
\text { (880 to } 1000)\end{array}$ & & & & \\
\hline Adverse events & See comment & See comment & Not estimable & $\begin{array}{l}0 \\
\text { (1 study) }\end{array}$ & See comment & $\begin{array}{l}12 \text { adverse events reported in the NPWT } \\
\text { group and } 7 \text { events in the standard care } \\
\text { group. The methods of data collection } \\
\text { and analysis were limited }\end{array}$ \\
\hline
\end{tabular}

${ }^{*}$ The basis for the assumed risk (e.g. the median control group risk across studies) is provided in footnotes. The corresponding risk (and its $95 \%$ confidence interval) is based on the assumed risk in the comparison group and the relative effect of the intervention (and its $95 \% \mathrm{Cl}$ ).

$\mathrm{Cl}$ : confidence interval; HR: hazard ratio; NPWT: negative pressure wound therapy RR: risk ratio

GRADE Working Group grades of evidence

High quality: Further research is very unlikely to change our confidence in the estimate of effect

Moderate quality: Further research is likely to have an important impact on our confidence in the estimate of effect and may change the estimate

Low quality: Further research is very likely to have an important impact on our confidence in the estimate of effect and is likely to change the estimate 


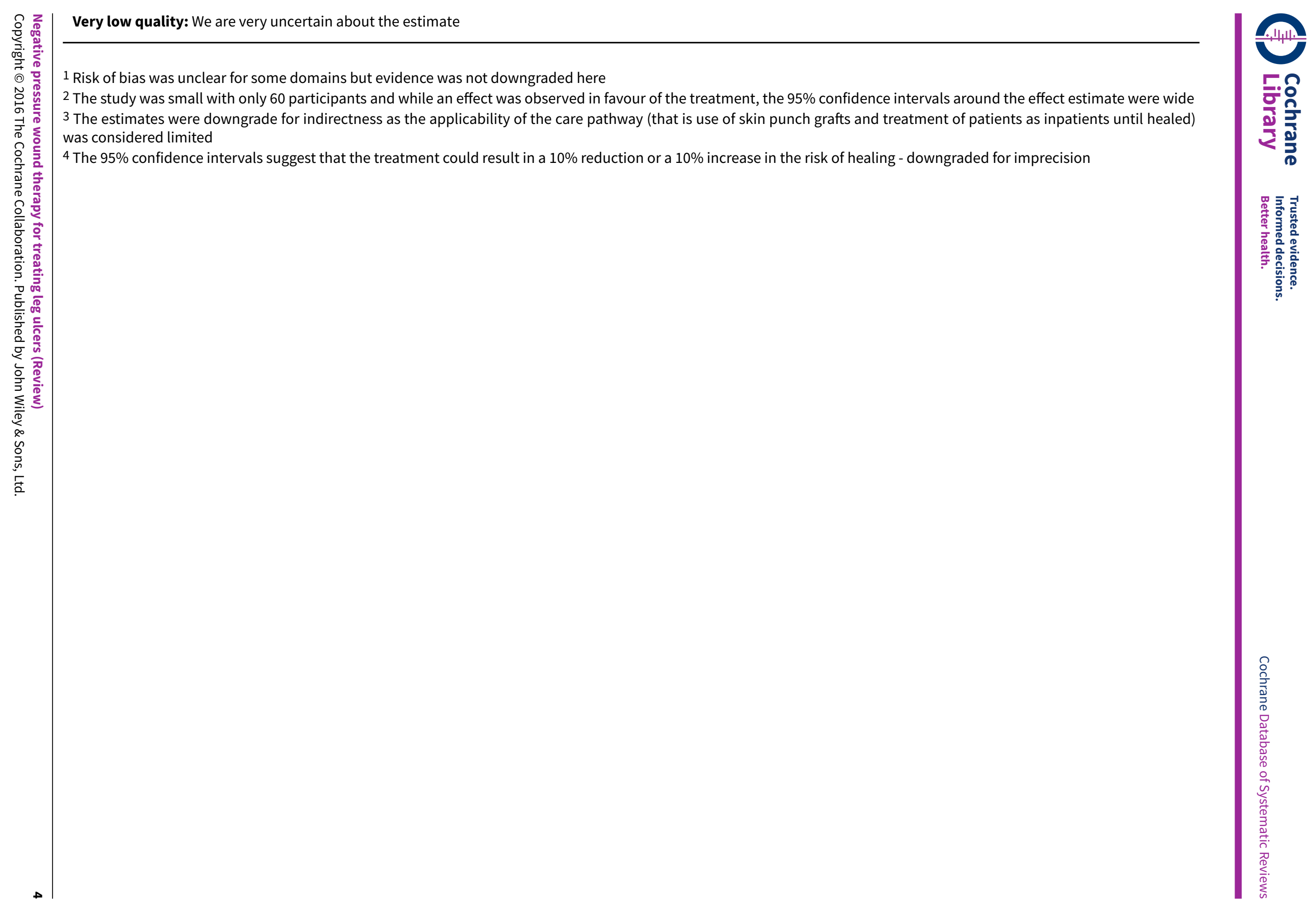




\section{B A C K G R O U N D}

\section{Description of the condition}

Leg ulcers are open, skin wounds on the lower leg (between the knee and the ankle) that can last weeks, months, or even years, and occur as a consequence of arterial or valvular insufficiency. Structural problems with leg veins (such as damage to the valves or vein blockage) are the most common cause of leg ulceration and result in venous leg ulcers. These problems mean that blood no longer returns efficiently from the legs towards the heart and the pressure within the leg veins rises (Ghauri 2010). The precise chain of events that links high venous pressures with skin breakdown and subsequent chronic wounds is not fully understood (Coleridge 1988). Leg ulcers are frequently associated with a combination of venous disease and vascular disease (which impairs arterial blood supply); such ulcers are said to have a 'mixed aetiology'. Open skin ulceration that is due solely to limb ischaemia (a lack of oxygen reaching the leg tissues) is less common. Accurate, current estimates of leg ulcer prevalence are hard to identify because most surveys do not differentiate between causes of leg ulceration, or do so for each limb but not for each patient (Moffatt 2004; Srinivasaiah 2007; Vowden 2009). Estimates of the prevalence of open leg ulceration (from any cause) range from 0.4 cases per 1000 (Walker 2002), to 4.8 cases per 1000 (Johnson 1995). A recent estimate suggests that venous ulceration has a prevalence of 0.29 cases per 1000 in the UK, whilst mixed arterial/venous leg ulceration has a prevalence of 0.11 per 1000 (Cullum 2014).

Venous disease is a chronic condition that is characterised by periods of ulceration (i.e. an open wound) followed by healing and then recurrence, although published contemporary data are lacking (Callam 1987). An early cross-sectional survey reported that half of current or recent ulcers had been open for up to nine months and that $35 \%$ of people with leg ulcers had experienced four or more episodes (Callam 1987). Cohort data from 20,000 people have shown that initial wound area and duration accurately predict healing (Margolis 2004). Ulcers smaller than $10 \mathrm{~cm}^{2}$ and that have existed for less than 12 months when first reported to a doctor have a $29 \%$ risk of not healing by the 24th week of care, whilst ulcers that exceed $10 \mathrm{~cm}^{2}$ and have existed for longer than 12 months before being reported have a $78 \%$ chance of not healing by 24 weeks (Margolis 2004).

The first line treatment for venous leg ulcers is compression therapy in the form of bandages, stockings or mechanical devices (O'Meara 2012). This application of external pressure around the lower leg assists venous return and reduces venous reflux (Woo 2013). Alongside compression, wound dressings are almost always applied to open ulcers. The primary rationale for using a dressing is to protect the surface of the ulcer, however other considerations such as absorption of exudate or antimicrobial properties also play a role in treatment selection. Other treatments for venous leg ulcers include venous surgery (removal of incompetent superficial veins; Gohel 2007), and drugs such as pentoxifylline (Jull 2012).

Differential diagnosis of the type of leg ulcer (i.e. the underlying cause) is made by taking a clinical history, physical examination, laboratory tests and haemodynamic assessment (RCN 2006; SIGN 2010). The latter typically includes an assessment of arterial supply to the leg using the ankle brachial pressure index (ABPI), measured using a hand-held Doppler ultrasound scanner.
Leg ulcers are associated with considerable cost to patients and to healthcare providers. Two systematic reviews summarised the literature on health-related quality of life in patients with leg ulcers (Persoon 2004; Herber 2007). Both included qualitative and quantitative evaluations and reported that the presence of leg ulceration was associated with pain, restriction of work and leisure activities, impaired mobility, sleep disturbance, reduced psychological well-being and social isolation. Recent research suggests that people with complex wounds, including those with venous leg ulcers, commonly see complete wound healing as the most important outcome (Madden 2014).

The cost of treating an open venous leg ulcer in the UK has been estimated to be around GBP 1700 per year at 2012 prices (Ashby 2014). A large part of ulcer treatment cost comprises nursing time. A study in Bradford, UK (population approximately 500,000) estimated that for the financial year 2006 to 2007, GBP 1.69 million was spent on dressings and compression bandages and GBP 3.08 million on nursing time (estimates derived from resource use data for all wound types, not just venous leg ulcers; Vowden 2009). We were unable to identify additional, contemporary, international cost data.

\section{Description of the intervention}

Negative pressure wound therapy (NPWT) is a device-based treatment used widely in wound care that is promoted for use on complex wounds (Guy 2012). NPWT can be used in hospitals and in patients' homes. NPWT involves the application of a wound dressing by a health professional through which a negative pressure (or vacuum) is applied, often with any wound and tissue fluid that is drawn away from the area being collected in a canister. The intervention was developed in the 1990s, and the uptake of NPWT in the healthcare systems of developed countries has been dramatic. A US Department of Health report estimated that between 2001 and 2007, Medicare payments for NPWT pumps and associated equipment increased from USD 24 million to USD 164 million (an increase of almost 600\%; Department of Health and Human Services 2009). Initially only one NPWT manufacturer supplied NPWT machines (the VAC system: $\mathrm{KCl}$, San Antonio Texas), however, as the NPWT market has grown, a number of different commercial NPWT systems have been developed, with machines becoming smaller and more portable. Indeed, the most recent introduction to the market is a single use, or 'disposable', negative pressure product. Ad hoc, non-commercial, negative pressure devices are also used, especially in resource-poor settings. These devices tend to use simple wound dressings, such as gauze, or transparent occlusive (non-permeable) dressings, with negative pressure generated in hospital by vacuum suction pumps.

A number of different healthcare professionals prescribe and apply NPWT, and it is now used both in secondary and primary (community) care, particularly following the introduction of ambulatory systems. Whilst the NPWT systems outlined above differ in a number of respects - such as type of pressure (constant or cyclical) applied to the wound, the material in contact with the surface of the wound and also the type of dressing used - the principle of applying a negative pressure to the wound in a closed environment is the same for all products. 


\section{How the intervention might work}

NPWT collects high volumes of wound exudate and is thought to reduce the frequency of dressing changes by keeping anatomicallychallenging wounds clean, and reducing odour. It is also suggested that the application of suction to the wound promotes healing by the drawing together of wound edges, increasing perfusion, and removing infectious material and exudate $(\mathrm{KCl} 2012$; Huang 2014). NPWT might exert a beneficial effect through preventing unnecessary dressing changes and repeated exposure of the wound to the environment.

Potential negative outcomes from NPWT include wound maceration (softening due to exposure to liquid), retention of dressings, and wound infection, as well as other injuries (FDA 2011). NPWT devices are usually worn continually by patients during treatment. They can interfere with mobility, and, anecdotally, are often noisy, which prevents some patients from sleeping.

\section{Why it is important to do this review}

Given its widespread use, it is important to assess the current evidence regarding the clinical and cost effectiveness of NPWT. The UK Scottish Intercollegiate Guidelines Network (SIGN) guidelines note that there is insufficient evidence on which to base a recommendation for use of NPWT in cases of chronic venous leg ulcers (SIGN 2010). The production of a robust and updated systematic review will contribute by identifying, appraising and synthesising the current evidence base to inform decision makers and possibly guide future research. This review is part of a suite of reviews that will look at the effectiveness of NPWT on wounds (Dumville 2014).

\section{O B J E C T IVES}

To assess the effects of negative pressure wound therapy (NPWT) for treating leg ulcers in any care setting.

\section{METHODS}

\section{Criteria for considering studies for this review}

\section{Types of studies}

We included published and unpublished randomized controlled trials (RCTs), including cluster RCTs, irrespective of language of report. Cross-over trials were only included if they reported outcome data at the end of the first treatment period and before cross over. Studies using quasi-randomisation were excluded.

\section{Types of participants}

We included RCTs that recruited people described in the primary report as having leg ulcers, managed in any care setting. As the method of defining ulceration could vary, we accepted the definitions used by the study authors.

\section{Types of interventions}

The primary intervention of interest was NPWT (both commercial and non-commercial treatments). We included any RCT in which use of a specific NPWT machine during the treatment period was the only systematic difference between treatment groups. We anticipated that likely comparisons would include use of NPWT during the care pathway compared with no use of NPWT, or comparison of different types/brands of NPWT use during the care pathway.

\section{Types of outcome measures}

We list primary and secondary outcomes below. If a study was otherwise eligible (i.e. correct study design, population and intervention/comparator) but did not report a listed outcome, then we contacted the study authors where possible to establish whether an outcome of interest to the review had been measured but not reported.

We reported outcome measures at the latest time point available (assumed to be length of follow-up if not specified) and the time point specified in the methods as being of primary interest (if this was different from the latest time point available). For all outcomes we classed assessment of outcome measures from:

- under one week to eight weeks as short term;

- over eight weeks to 26 weeks as medium term; and

- over 26 weeks as long term.

\section{Primary outcomes}

The primary outcomes for this review were complete wound healing and adverse events.

\section{Complete wound healing}

For this review we regarded the following as providing the most relevant and rigorous measures of outcome:

- time to complete wound healing: we planned to record whether this has been correctly analyzed using censored data and with adjustment for prognostic covariates such as baseline size;

- the proportion of ulcers healed (frequency of complete healing).

Where both of the outcomes above have been reported we present all data, but regarded time to healing as being the preferred outcome. We accepted authors' definitions of what constituted a healed wound.

\section{Adverse events}

We extracted reported data on adverse events and classed them as 'serious adverse events' and 'non-serious adverse events' where a clear methodology for the collection of adverse event data was provided. This methodology needed to make it clear whether events were reported at the participant level or, where multiple events/person were reported, that an appropriate adjustment had been made for data clustering. We did not extract individual types of adverse events such as pain or infection, that require specific assessment, under this outcome - rather this was the assessment of any event classed as adverse by the patient and or health professional during the trial.

\section{Secondary outcomes}

- Participant health-related quality of life/health status (measured using a standardised generic questionnaire such as EQ-5D, SF-36, SF-12 or SF-6 or wound-specific questionnaires such as the Cardiff wound impact schedule). We will not include ad hoc measures of quality of life that are not likely to be validated and would not be common to multiple trials. 
- Resource use: including measurements of resource use such as number of dressing changes, nurse visits, length of hospital stay and re-operation/intervention.

- Costs: any costs applied to resource use.

- Wound recurrence.

- Wound infection: as defined by author.

- Mean pain scores: (including pain at dressing change) will be included only where it is reported as either a presence or absence of pain or as a continuous outcome using a validated scale such as a visual analogue scale (VAS).

- Proportion of wounds closed with surgery or time to preparation for surgery: complete wound closure (including skin grafting) that was the result of surgical closure rather than healing or time to readiness for surgery.

\section{Search methods for identification of studies}

\section{Electronic searches}

We searched the following electronic databases to retrieve reports of relevant RCTs:

- the Cochrane Wounds Group Specialised Register (searched 21 May 2015);

- the Cochrane Central Register of Controlled Trials (CENTRAL; The Cochrane Library 2015, Issue 4);

- Ovid MEDLINE (1946 to 20 May 2015);

- Ovid MEDLINE (In-Process \& Other Non-Indexed Citations 20 May 2015);

- Ovid EMBASE (1974 to 20 May 2015);

- EBSCO CINAHL (1982 to 21 May 2015)

The CENTRAL search string is given below.

\#1 MeSH descriptor: [Negative-Pressure Wound Therapy] explode all trees

\#2 MeSH descriptor: [Suction] explode all trees

\#3 MeSH descriptor: [Vacuum] explode all trees

\#4 ("negative pressure" or negative-pressure or TNP or NPWT):ti,ab,kw

\#5 (sub-atmospheric or subatmospheric):ti,ab,kw

\#6 ((seal ${ }^{\star}$ next surface $\left.{ }^{\star}\right)$ or (seal ${ }^{\star}$ next aspirat $\left.\left.{ }^{\star}\right)\right):$ ti,ab,kw

\#7 (wound near/3 suction*):ti,ab,kw

\#8 (wound near/3 drainage):ti,ab,kw

\#9 (foam next suction) or (suction next dressing ${ }^{\star}$ ):ti,ab,kw

\#10 (vacuum assisted closure or VAC):ti,ab,kw

\#11 (vacuum next therapy) or (vacuum next dressing*) or (vacuum next seal $\left.{ }^{\star}\right)$ or (vacuum next assist* ${ }^{\star}$ or (vacuum near closure) or (vacuum next compression) or (vacuum next pack*) or (vacuum next drainage) or (suction* adj drainage):ti,ab,kw

$\# 12$ (\#1 or \#2 or \#3 or \#4 or \#5 or \#6 or \#7 or \#8 or \#9 or \#10 or \#11)

\#13 MeSH descriptor: [Leg Ulcer] explode all trees

\#14 ((varicose next ulcer ${ }^{\star}$ ) or (venous next ulcer*) or (leg next ulcer $\left.{ }^{\star}\right)$ or (stasis next ulcer ${ }^{\star}$ ) or (crural next ulcer*) or "ulcus cruris" or "ulcer* cruris"):ti,ab,kw

\#15 \#13 or \#14

$\# 16 \# 12$ and \#15

We combined the Ovid MEDLINE search with the Cochrane Highly Sensitive Search Strategy for identifying randomized trials in MEDLINE: sensitivity- and precision-maximising version (2008 revision; Lefebvre 2011). We combined the EMBASE search with the Ovid EMBASE filter developed by the UK Cochrane Centre (Lefebvre 2011). We combined the CINAHL search with the trial filters developed by the Scottish Intercollegiate Guidelines Network (SIGN 2012). There were no restrictions with respect to language, date of publication or study setting. We also searched the following clinical trials registries:

- ClinicalTrials.gov (www.clinicaltrials.gov);

- WHO International Clinical Trials Registry Platform (apps.who.int/trialsearch/Default.aspx);

- EU Clinical Trials Register (www.clinicaltrialsregister.eu).

\section{Searching other resources}

We contacted corresponding authors and the manufacturers and distributors of NPWT. We tried to identify other potentially eligible trials or ancillary publications by searching the reference lists of included trials as well as relevant systematic reviews, metaanalyses, and health technology assessment reports.

\section{Data collection and analysis}

\section{Selection of studies}

Two review authors independently assessed the titles and abstracts of the citations retrieved by the searches for relevance. After this initial assessment, we obtained full text copies of all studies considered to be potentially relevant. Two review authors independently checked the full papers for eligibility; we planned to resolve disagreements through discussion with, where required, the input of a third review author. Where required and possible, we contacted study authors where the eligibility of a study was unclear. We recorded all reasons for exclusion of studies for which we had obtained full copies of the text. We have completed a PRISMA flowchart to summarize this process (Liberati 2009).

Where studies were reported in multiple publications/reports, we obtained all publications. Whilst the study was included only once in the review, data were extracted from all reports to ensure we obtained maximal relevant data.

\section{Data extraction and management}

We extracted and summarize details of the eligible studies. Two review authors extracted data independently and resolved disagreements by discussion, drawing on a third review author where required. Where data were missing from reports, we attempted to contact the study authors to obtain this information. Where a study with more than two intervention arms was included, we planned to only extract data from intervention and control groups that met the eligibility criteria.

We extracted the following data where possible by treatment group for the pre-specified interventions and outcomes in this review:

- country of origin;

- type of wound and surgery;

- unit of randomisation (per patient) - single wound or multiple wounds on the same patient;

- unit of analysis;

- trial design e.g. parallel, cluster;

- care setting;

- number of participants randomized to each trial arm; 
- eligibility criteria and key baseline participant data;

- details of treatment regimen received by each group;

- duration of treatment;

- details of any co-interventions;

- primary and secondary outcome(s) (with definitions);

- outcome data for primary and secondary outcomes (by group);

- duration of follow-up;

- number of withdrawals (by group);

- publication status of study; and

- source of funding for trial.

We collected outcome data for relevant time points as described in Types of outcome measures.

\section{Assessment of risk of bias in included studies}

Two review authors independently assessed the included studies for risk of bias using the Cochrane Collaboration tool for assessing risk of bias (Higgins 2011a). This tool addresses six specific domains: sequence generation, allocation concealment, blinding, incomplete data, selective outcome reporting and other issues. In this review we recorded issues with unit of analysis, for example where a cluster trial has been undertaken but analyzed at the individual level in the study report (Appendix 1). We assessed blinding and completeness of outcome data for each of the review outcomes separately. We note that, since wound healing is a subjective outcome, it can be at high risk of measurement bias when outcome assessment is not blinded. We have presented our assessment of risk of bias using two 'Risk of bias' summary figures; one is a summary of bias for each item across all studies, and the other shows a cross-tabulation of each trial by all of the risk of bias items. In future, we will class studies with an assessment of high risk of bias for the randomisation sequence domain or the allocation concealment domain, or both, to be at high risk of selection bias; those at high risk of bias for the blinded outcome assessment domain (for a specified outcome) to be at a high risk of detection bias; and those with a high risk of bias due to incomplete data to be at a high risk of attrition bias (for specified outcome).

For trials using cluster randomisation, we planned to assessed the risk of bias considering recruitment bias, baseline imbalance, loss of clusters, incorrect analysis and comparability with individually randomized trials (Higgins 2011b; Appendix 2).

\section{Measures of treatment effect}

For dichotomous outcomes we calculated the risk ratio (RR) with $95 \%$ confidence intervals $(\mathrm{CI})$. For continuously distributed outcome data we used the mean difference (MD) with 95\% Cls when all trials used the same assessment scale. When trials use different assessment scales, we will use the standardised mean difference (SMD) with $95 \% \mathrm{Cls}$. We only considered mean or median time to healing without survival analysis as a valid outcome if reports specified that all wounds healed (i.e. if the trial authors regarded time to healing as a continuous measure as there is no censoring). Time-to-event data (e.g. time-to-complete wound healing), was reported as hazard ratios (HR) where possible in accordance with the methods described in the Cochrane Handbook for Systematic Reviews of Interventions (Deeks 2011). If studies reporting time-toevent data (e.g. time to healing) did not report a hazard ratio, then, where feasible, we planned to estimate this using other reported

Negative pressure wound therapy for treating leg ulcers (Review)

Copyright $\odot 2016$ The Cochrane Collaboration. Published by John Wiley \& Sons, Ltd. outcomes, such as the numbers of events, through the application of available statistical methods (Parmar 1998).

\section{Unit of analysis issues}

Where studies randomized at the participant level and measured outcomes at the wound level, for example for wound healing, and the number of wounds appeared to be equal to the number of participants, we treated the participant as the unit of analysis.

We had anticipated a possible unit of analysis issue if individual participants with multiple wounds were randomized, the allocated treatment was used on the multiple wounds per participant (or perhaps only on some participants) and then data presented and analyzed by wound not person. This is a type of clustered data and presents a unit of analysis error that inflates precision. In cases where included studies contain some or all clustered data we planned to report this alongside whether data had been (incorrectly) treated as independent. We recorded this as part of the risk of bias assessment. We did not plan to undertake further calculation to adjust for clustering.

\section{Dealing with missing data}

It is common to have data missing from trial reports. Excluding participants post-randomisation from the analysis, or ignoring those participants who are lost to follow-up compromises the randomisation, and potentially introduces bias into the trial. Where there were missing data we thought should be included in the analyses, we contacted relevant study authors to enquire whether these data were available.

Where data remained missing for 'proportion of wounds healed', for analysis we assumed that if randomized participants were not included in an analysis, their wound did not heal (i.e. they would be considered in the denominator but not the numerator).

In a time-to-healing analysis using survival analysis methods, dropouts should be accounted for as censored data so we took no action regarding missing data.

For continuous variables, for example, length of hospital stay and for all secondary outcomes, we presented available data from the study reports/study authors and did not anticipate imputing missing data. Where measures of variance were missing we calculated these where possible. If calculation was not possible we contacted the study authors. Should these measures of variation not be available, the study will be excluded from any relevant metaanalyses conducted.

\section{Assessment of heterogeneity}

Assessment of heterogeneity can be a complex, multi-faceted process. Firstly, we planned to consider clinical and methodological heterogeneity: that is the degree to which the included studies varied in terms of participant, intervention, outcome and characteristics such as length of follow-up. This assessment of clinical and methodological heterogeneity would be supplemented by information regarding statistical heterogeneity - assessed using the $\mathrm{Chi}^{2}$ test (we would consider a significance level of $\mathrm{P}<0.10$ to indicate statistically significant heterogeneity) in conjunction with the $I^{2}$ measure (Higgins 2003). $I^{2}$ examines the percentage of total variation across RCTs that is due to heterogeneity rather than chance (Higgins 2003). Very broadly, we consider that $\mathrm{I}^{2}$ values of $25 \%$ or less may mean a low level of heterogeneity (Higgins 2003), 
and values of $75 \%$ or more indicate very high heterogeneity (Deeks 2011). Where there is evidence of high heterogeneity we anticipated exploring this further where possible: see Data synthesis.

\section{Assessment of reporting biases}

Reporting biases arise when the dissemination of research findings is influenced by the nature and direction of results. Publication bias is one of a number of possible causes of 'small study effects', that is, a tendency for estimates of the intervention effect to be more beneficial in smaller RCTs. Funnel plots allow a visual assessment of whether small study effects may be present in a meta-analysis. A funnel plot is a simple scatter plot of the intervention effect estimates from individual RCTs against some measure of each trial's size or precision (Sterne 2011). We planned to present funnel plots for meta-analyses comprising 10 RCTs or more using RevMan 5.3 (RevMan 2014).

\section{Data synthesis}

Where possible we planned to combine details of included studies in a narrative review according to type of comparator, possibly by location of/type of wound and then by outcomes by time period. We planned to assess clinical and methodological heterogeneity and anticipated pooling data when studies appeared appropriately similar in terms of wound type, intervention type, duration of follow-up and outcome type.

In terms of meta-analytical approach, in the presence of clinical heterogeneity (review author judgement) or evidence of statistical heterogeneity, or both, we planned to use the random-effects model. We planned to use a fixed-effect approach only when clinical heterogeneity was thought to be minimal and statistical heterogeneity was estimated as non-statistically significant for the $\mathrm{Chi}^{2}$ value and $0 \%$ for the $\mathrm{I}^{2}$ assessment (Kontopantelis 2012). This approach was adopted as it is recognised that statistical assessments can miss potentially important betweenstudy heterogeneity in small samples, hence the preference for the more conservative random-effects model (Kontopantelis 2013). Where clinical heterogeneity was thought to be acceptable, or of interest, we planned to meta-analyse even when statistical heterogeneity was high, attempting to interpret the causes behind this heterogeneity. We anticipated use of meta-regression for this purpose, if possible (Thompson 1999).

We presented data using forest plots where possible. For dichotomous outcomes we present the summary estimate as a risk ratio (RR) with $95 \% \mathrm{Cl}$. Where continuous outcomes were measured in the same way across studies, we planned to present a pooled mean difference (MD) with $95 \% \mathrm{Cl}$; we planned to pool standardised mean difference (SMD) estimates where studies measured the same outcome using different methods. For time-to-event data, we planned to plot (and, if appropriate, pool) estimates of hazard ratios and $95 \% \mathrm{Cls}$ as presented in the study reports using the generic inverse variance method in RevMan 5.3. Where time to healing was analyzed as a continuous measure, but it was not clear if all wounds healed, we planned to document use of the outcome in the study but data were not to be summarised or used in any meta-analysis.

We planned to obtain pooled estimates of treatment effect using Cochrane RevMan software version 5.3 (RevMan 2014).

\section{'Summary of findings' tables}

We presented the main results of the review in 'Summary of findings' tables. These tables present key information concerning the quality of the evidence, the magnitude of the effects of the interventions examined, and the sum of the available data for the main outcomes (Schunemann 2011a). The 'Summary of findings' tables also include an overall grading of the evidence related to each of the main outcomes using the GRADE (Grades of Recommendation, Assessment, Development and Evaluation) approach. The GRADE approach defines the quality of a body of evidence as the extent to which one can be confident that an estimate of effect or association is close to the true quantity of specific interest. The quality of a body of evidence involves consideration of within-trial risk of bias (methodological quality), directness of evidence, heterogeneity, precision of effect estimates and risk of publication bias (Schunemann 2011b). We planned to present the following outcomes in the 'Summary of findings' tables:

- time to complete ulcer healing where analyzed using appropriate survival analysis methods and/or;

- proportion of ulcers completely healing during the trial period;

- adverse events.

\section{Subgroup analysis and investigation of heterogeneity}

Where there was evidence of between-trial heterogeneity and where it was feasible we envisaged a subgroup analysis being conducted for:

- type of negative pressure system being used;

- studies at low risk of selection bias versus those at unclear or high risk.

\section{RES U LTS}

\section{Description of studies}

\section{Results of the search}

See Figure 1 for a summary of the results of our search. 
Figure 1. Study flow diagram

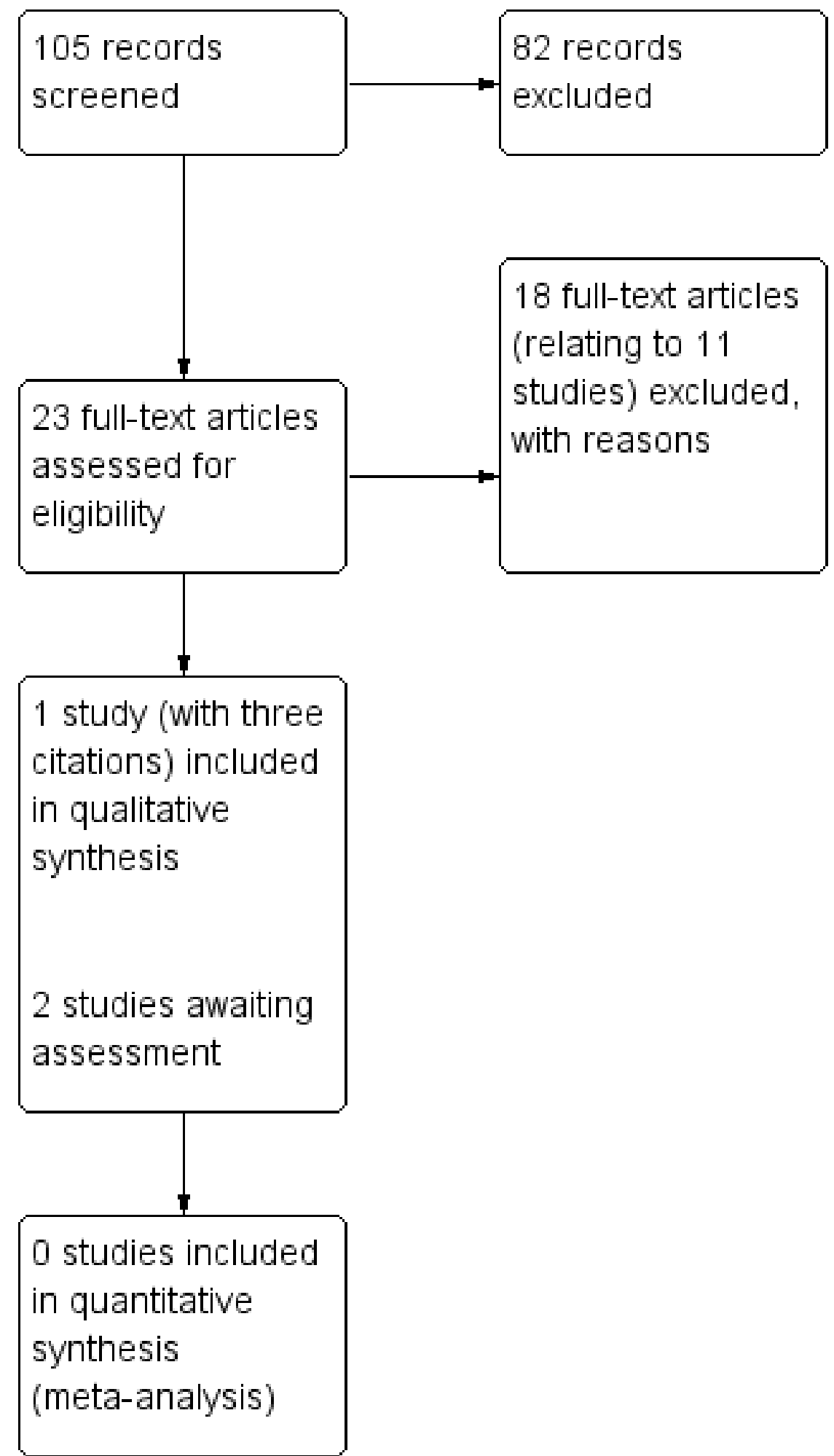

Searches identified 105 citations for this review. In total 23 citations relating to 14 studies were obtained as full text. Of these 11 studies were excluded from the review (see Excluded studies), two studies are awaiting assessment (Pruksapong 2011; Marston 2015), and one study was included in the review (Vuerstaek 2006). We did not identify any relevant on-going studies (Registers checked 16th May 2015 ). 


\section{Included studies}

This review include one study that was conducted in an inpatient care setting in the Netherlands (Vuerstaek 2006). The study randomized 60 participants with leg ulcers: $43 \%$ of participants had venous leg ulcers, $43 \%$ had leg ulcers that were arteriolosclerotic in origin and $13 \%$ had mixed venous/arterial ulcers. To be eligible for entry into the study, participants had to have recalcitrant ulcers that remained unhealed after "extensive (6 months) treatment in an outpatient clinic according to SIGN guidelines had failed." (Vuerstaek 2006).

The included study compared NPWT with standard care in the treatment of these hard-to-heal leg ulcers. All study ulcers received treatment with a punch skin-graft transplant preceded and followed by application or no application of NPWT. Wounds allocated to the NPWT group received continuous negative pressure until $100 \%$ granulation (wound preparation stage) was achieved. A punch skin-graft transplant was then conducted and it seems that the wound was exposed to further negative pressure therapy for four days and then standard care. Participants allocated to the control arm received standard care with dressings and compression until $100 \%$ granulation. These participants also received a punch skin-graft transplant and then further treatment with standard care.

The study protocol stated that all participants remained as hospital in-patients until their wounds had healed.

\section{Excluded studies}

In total we obtained the full text for 11 studies and then excluded them from this review for the following reasons:

- study population had a range of wounds - unable to isolate leg ulcer data (Joseph 2000; Moues 2005; Braakenburg 2006; Hu 2009; Perez 2010; Armstrong 2012; Rahmanian Schwarz 2012);

- not relevant wound type (de Laat 2011);

- no relevant outcomes (Lantis 2004; Dini 2009) - we made attempts to contact authors;

- not a randomized controlled trial (Tauro 2007).

\section{Risk of bias in included studies}

See Characteristics of included studies for the risk of bias table, and Figure 2 for a risk of bias summary, for the included study.

Figure 2. Risk of bias summary: review authors' judgements about each risk of bias item for each included study

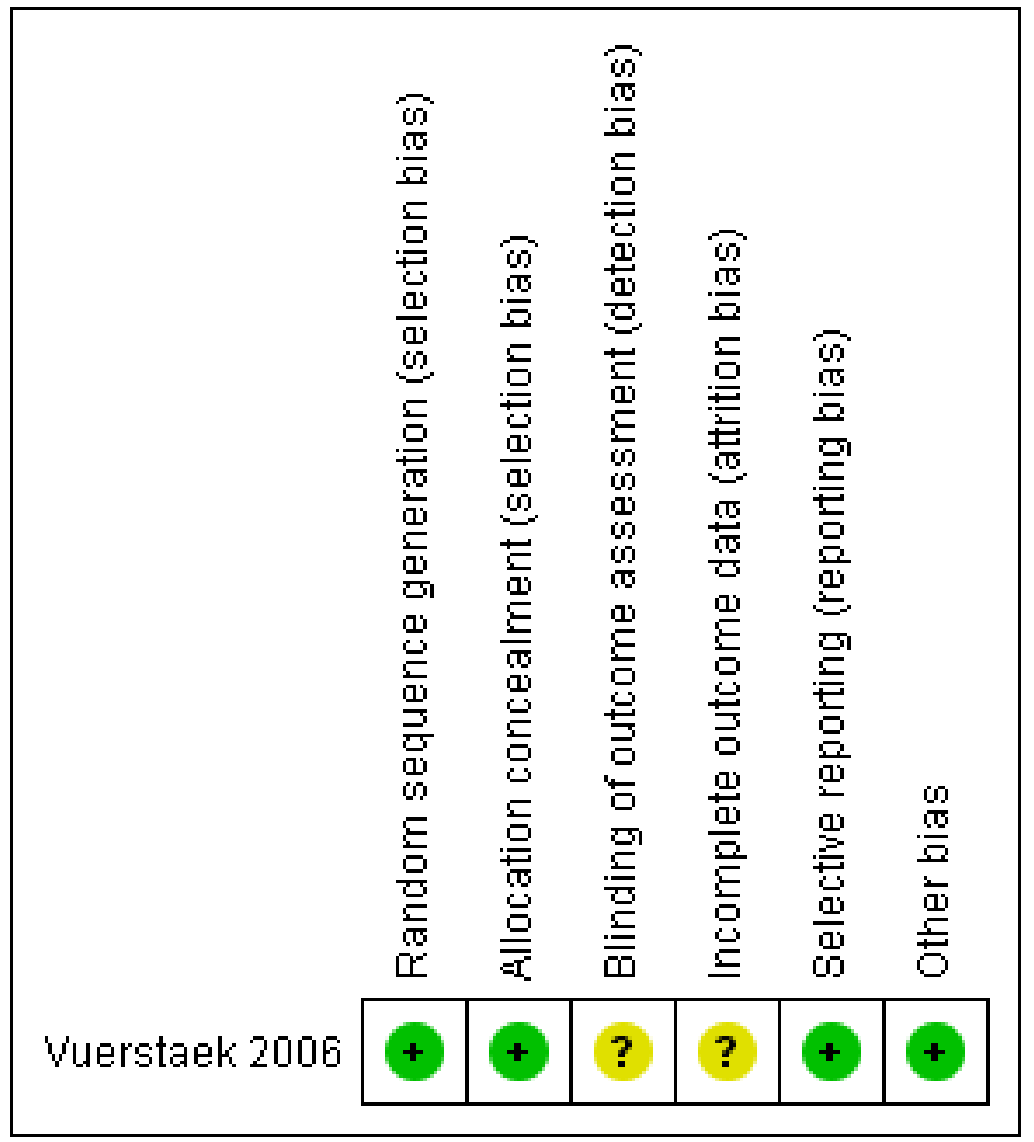

\section{Allocation}

The included study used a computer-generated randomisation sequence and central allocation. It was deemed to be at low risk of selection bias.

\section{Incomplete outcome data}

In total six participants may have been excluded from the analysis, this was $10 \%$ of the total study population. We deemed the study to be at unclear risk of attrition bias. 


\section{Selective reporting}

We deemed the study to be at low risk of reporting bias.

\section{Other potential sources of bias}

We noted no other potential sources of bias, such as unit of analysis issues.

\section{Effects of interventions}

See: Summary of findings for the main comparison Negative pressure wound therapy compared to standard care for people with leg ulcers undergoing a punch skin-graft

See Table 1 for study and study outcome data.

\section{Comparison 1: negative pressure wound therapy (NPWT) compared with standard care (in patients undergoing a punch skin-graft transplant)}

We included one study $(n=60)$ in this comparison (Vuerstaek 2006). The participants had hard-to-heal ulcers. The study compared NPWT followed by a punch skin-graft transplant and further NPWT treatment with standard wound care followed by a punch skingraft transplant and subsequent standard care. Wound healing was defined as $100 \%$ epithelialisation. We deemed the study to be at unclear risk of detection and attrition bias, and at low risk for selection, reporting outcome and other biases.

\section{Primary outcome: complete wound healing}

The median time to healing in the NPWT group was 28 days ( $95 \%$ $\mathrm{Cl} 25.5$ to 32.5 ) compared with 45 days ( $95 \% \mathrm{Cl} 36.2$ to 53.8 ) in the standard care group. The study reported an adjusted hazard ratio of $3.2,95 \% \mathrm{Cl} 1.7$ to 6.2 (adjusted for ulcer area; smoking; infection signs; ulcer history; therapy) suggesting a higher hazard (chance) of healing with NPWT. GRADE assessment: low quality evidence (due to imprecision and indirectness): Summary of findings for the main comparison.

The study reported that all but one participant in each group healed - as laid out in our Dealing with missing data section, we assumed that any participants for whom complete healing data were missing $(n=6)$ did not heal. This approach meant that $97 \%$ (29/30) of participants healed in both groups (RR 1.00, 95\% Cl 0.91 to 1.10; Analysis 1.1). The time period over which this proportion of healing occurred was not clear, but time-to-event data suggested that all or almost all participants were healed after 80 days from randomisation. GRADE assessment: low quality evidence (due to imprecision and indirectness): Summary of findings for the main comparison.

\section{Primary outcome: adverse events}

Twelve adverse events were reported in the NPWT group compared with seven in the standard care group. The methods of adverse events data collection were not reported, and it was not clear whether data were reported per participant. These data have not been analyzed further.

\section{Secondary outcome: participant health-related quality of life/ health status}

The study reported an EQ-5D score at eight weeks - the EQ-5D instrument is a validated tool for assessing health utility that measures self-reported responses from five domains relating to health. There was no evidence of a difference in EQ-5D scores at eight weeks between study groups: mean difference $1.00(95 \% \mathrm{Cl}$ -6.88 to 8.88 ; Analysis 1.2). There was uncertainty in this estimate as the study was small and the intervals around the mean difference ranged from an EQ-5D score seven points lower in the NPWT group to nearly nine points higher. GRADE assessment: low quality evidence due to very serious imprecision and the applicability of the delivery of the intervention to most patients. The ulcers here were hard-to-heal, which is the case in a sub-population of leg ulcer patients. In addition to this the use of punch skin-graft transplantation as a treatment for leg ulcers is potentially limited and all participants were treated as in-patients until healing, which is also largely atypical.

\section{Secondary outcome: costs}

The study reported that costs for personnel time and the material costs for wound care procedures until complete healing were recorded, but even though participants were kept as in-patients, hospital costs were not included. The mean cost of treatment in the NPWT group was USD 3881 compared with USD 5452 in the standard care group. No measure of variation around these estimates was reported and further analysis here was not possible.The study reported this difference as being statistically significant, but we are unable to reproduce this analysis with the available data.

\section{Secondary outcome: wound recurrence}

There were 12 incidences of ulcer recurrence in the NPWT group compared with 10 in the standard care group: these were reported in the study as being $52 \%$ and $42 \%$ of the group populations, respectively. It is not clear what the denominator was (that is the number healed at the time recurrence was assessed) and the data have not been analyzed further. The study reported that the median time from healing to recurrence was four months in the NPWT and two months in the standard care group, no other data were presented and these data cannot be analyzed further. It is also important to note that these recurrence data were not randomized as only those that had healed could recur.

\section{Secondary outcome: mean pain scores}

Pain scores at eight-week follow-up were universally low in both groups for the Short Form McGill Pain Questionnaire: both groups had a score of 1 (SD 1). Mean pain scores at eight weeks were also low, with no evidence of a difference between groups when measured with the Present Pain Intensity Instrument: scores were 0.2 (SD 0.7) in the NPWT group and 0.4 (SD 0.6) in the standard care group (mean difference $-0.20,95 \% \mathrm{Cl}-0.53$ to 0.13 ; Analysis 1.3 ). The size of the difference here was small. GRADE assessment: low quality evidence - due to some imprecision also indirectness relating to the population and the context in which the intervention was delivered.

\section{Secondary outcome: time to preparation for surgery}

The median time to preparation for surgery was seven days $(95 \% \mathrm{Cl}$ 5.7 to 8.3 ) in the NPWT group and 17 days ( $95 \% \mathrm{Cl} 10$ to 24$)$ in the standard care group. It seems that assessment of when to undergo surgery was made by people blinded to allocation, but this was not completely clear in the report and we have queried this with the author (awaiting response). The study authors recorded evidence of a difference between groups in time to wound preparation: hazard ratio 2.4 (95\% $\mathrm{Cl} 1.2$ to 4.7$)$. GRADE assessment: low quality 
evidence - due to some imprecision also indirectness relating to the population and the context in which the intervention was delivered. The ulcers were hard-to-heal, which is the case in a sub-population of leg ulcer patients. In addition to this, the use of punch skin-graft transplantation as a treatment for leg ulcers is potentially limited.

\section{Comparison 1: summary of NPWT compared with standard care}

There is some evidence from a small trial that use of NPWT in nonhealing wounds that undergo a punch skin-graft procedure can reduce time to healing. Some of this difference in time to healing might relate to faster wound bed preparation prior to surgery. The trial was undertaken in a study population with mixed ulcers, and most participants were treated as in-patients until healing occurred. NPWT was used as part of a treatment pathway for those with hard-to-heal leg ulcers in which wounds were prepared for a skin grafting procedure - it is likely that this will not be a common procedure for most leg ulcer patients in most settings.

\section{DISCUSSION}

\section{Summary of main results}

Data from one study $(n=60)$ compared negative pressure wound therapy (NPWT) with standard care for the treatment of hard to heal leg ulcers of various aetiologies. All ulcers in the study underwent a punch skin-graft transplantation and the trial treatments were used before and after the grafting. NPWT appeared to be used for four days postoperatively.

There was evidence of a reduction in time to healing in the NPWT group with an increase in the hazard (chance) of healing. These data were reported by study authors and not re-analysed in this review. GRADE assessment - low quality evidence.

The follow-up time for the study was unclear but it seems that most participants were followed up for a minimum of 12 months. There was no evidence of a difference in the number of ulcers healed during follow-up: GRADE assessment - low quality evidence.

There was also evidence of a difference in the time to wound preparation for grafting in the NPWT treated group: GRADE assessment - low quality evidence.

There was no evidence of a difference in the EQ-5D utility measure or pain scores at eight weeks between study groups, although some estimates were imprecise. GRADE assessment - low quality evidence.

\section{Overall completeness and applicability of evidence}

The use of NPWT for leg ulcers has only been evaluated in one study that specifically evaluated its use in the context of participants also receiving a punch skin-graft transplant. Thus the outcomes assessed can only be generalised to use of NPWT in this care pathway. There are currently no studies available that investigate the use of NPWT in other types of care pathways in the treatment of leg ulcers, such as those cared for at home (which is likely to be the majority of patients).

\section{Quality of the evidence}

Overall the evidence was of low quality, as estimates were imprecise; this occurred because this was a small study and so had limited power. The applicability of participants and interventions (in the context of the co-intervention used and the treatment of participants as in-patients until healing) was limited. The study was well conducted, although it did not report on the blinding procedures for outcome assessment clearly, which is very important in trials such as this where decisions on when surgery should be undertaken and on when a wound is healed have a subjective element.

\section{Potential biases in the review process}

The review considered as much evidence as it was possible to obtain, including studies that were not published in Englishlanguage journals. It is possible that there may be unpublished data that we have not been able to access, and there is a potential for publication bias that has been recognised previously in NPWT studies (Peinemann 2008).

\section{Agreements and disagreements with other studies or reviews}

The SIGN guidelines for chronic venous leg ulcers, which define venous leg ulcers as "an open lesion between the knee and the ankle joint that remains unhealed for at least four weeks and occurs in the presence of venous disease", included only this study when considering the use of NPWT (SIGN 2010). The guideline reports that there "is insufficient evidence on which to base a recommendation for TNP/VAC in chronic venous leg ulcer[sic]".

\section{AUTHORS' CONCLUSIONS}

\section{Implications for practice}

There is limited evidence regarding the use of negative pressure wound therapy (NPWT) for the treatment of leg ulcers, with only one small trial available that compared the use of NPWT with standard care before and after skin grafting. Given the current uncertainties, practitioners may elect to consider various characteristics such as costs and symptom management properties when choosing between alternative treatment options for leg ulcers.

\section{Implications for research}

Further research to evaluate the clinical and cost effectiveness of NPWT as a treatment for leg ulcers is warranted where it is a priority for patients, carers and health professionals. Large and robust RCTs would be the most appropriate study design, with the study population and setting of care being that most relevant to current practices and uncertainties. Studies that consider the cost and effects of NPWT would also provide useful data to inform decision making.

\section{ACKNOWLEDGEMENTS}

The authors would like to acknowledge the support of the Cochrane Wounds Group in devising this protocol, in particular Sally Bell-Syer for her help in refining the protocol and Amanda Briant for her help in devising the search strategy. In addition the authors are grateful to the following peer reviewers for their time and comments: Amanda Briant, Anne-Marie Glenny, Anne Lyddiatt, Brian Stafford, Dirk Ubbink and Karen Zulkowski; and also for the contribution of Megan Prictor, and Elizabeth Royle, copy-editors. 


\section{R E F E R E N C E S}

\section{References to studies included in this review}

\section{Vuerstaek 2006 \{published data only\}}

* Vuerstaek JDD, Vainas T, Wuite J, Nelemans P, Neumann MHA, Veraart JCJM. State-of-the-art treatment of chronic leg ulcers: a randomized controlled trial comparing vacuum-assisted closure (V.A.C) with modern wound dressings. Journal of Vascular Surgery 2006;44:1029-37.

Vuerstaek JDD, Veraart JCJ, Wuite J, Neumann HAM. The management of recalcitrant venous leg ulcers. Wound Repair and Regeneration 2003;11:A2.

Vuerstaek JDD, Wuite J, Neuman H, Steijlen P, Veraart JCJM. The management of recalcitrant leg ulcers. 2nd World Union of Wound Healing Societies Meeting; 2004 July 8-13; Paris. 2004.

\section{References to studies excluded from this review}

Armstrong 2012 \{published data only\}

* Armstrong DG, Marston WA, Reyzelman AM, Kirsner RS. Comparative effectiveness of mechanically and electrically powered negative pressure wound therapy devices: a multicenter randomized controlled trial. Wound Repair and Regeneration 2012;20(3):332-41.

Armstrong DG, Marston WA, Reyzelman AM, Kirsner RS. Comparison of negative pressure wound therapy with an ultraportable mechanically powered device vs. traditional electrically powered device for the treatment of chronic lower extremity ulcers: a multicenter randomized-controlled trial. Wound Repair and Regeneration 2011;19(2):173-80.

Armstrong DG, Marston WA, Reyzelman AM, Kirsner RS. NPWT comparative effectiveness trial. Wound Repair and Regeneration 2012;20(3):332-41.

\section{Braakenburg 2006 \{published data only\}}

Braakenburg A, Obdeijn MC, Feitz R, van Rooij IA, van Griethuysen AJ, Klinkenbijl JH. The clinical efficacy and cost effectiveness of the vacuum-assisted closure technique in the management of acute and chronic wounds: a randomized controlled trial. Plastic and Reconstructive Surgery 2006;118:390-400.

\section{de Laat 2011 \{published data only\}}

de Laat EH, van den Boogaard MH, Spauwen PH, van Kuppevelt DH, van Goor H, Schoonhoven L. Faster wound healing with topical negative pressure therapy in difficult-toheal wounds: a prospective randomized controlled trial. Annals of Plastic Surgery 2011;67:626-31.

\section{Dini 2009 \{published data only\}}

Dini V, Bertone MS, Romanelli M. Biochemical markers in venous leg ulcers during treatment with topical negative pressure. EWMA Journal 2009;2:Abstract 118.

\section{Hu 2009 \{published data only\}}

Hu KX, Zhang HW, Zhou F, Yao G, Shi JP, Wang LF. Observation on the therapeutic effects of negative-pressure wound therapy on the treatment of complicated and refractory wounds. Zhonghua Shao Shang Za Zhi [Chinese journal of burns] 2009;4:249-52.

\section{Joseph 2000 \{published data only\}}

Joseph E, Hamori CA, Bergman S, Roaf E, Swann NF, Anastasi GW. A prospective randomized trial of vacuum-assisted closure versus standard therapy of chronic nonhealing wounds. Wounds 2000;12:60-7.

\section{Lantis 2004 \{published data only\}}

Lantis JC, Gendics C. VAC therapy appears to facilitate STSG take when applied to venous leg ulcers. 2nd World Union of Wound Healing Societies Meeting; 2004 July 8-13; Paris. 2004; Vol. 87.

\section{Moues 2005 \{published data only\}}

Moues CM, Van Den Bemd GJCM, Heule F, Hovius SER. A prospective randomized trial comparing vacuum therapy to conventional moist gauze therapy. 2nd World Union of Wound Healing Societies Meeting; 2004 July 8-13; Paris. 2004; Vol. 87:A001.

Moues CM, Van Den Bemd GJCM, Meerding WJ, Hovius SER. Cost analysis comparing vacuum-assisted closure wound therapy to conventional moist gauze therapy. 2nd World Union of Wound Healing Societies Meeting; 2004 July 8-13; Paris. 2004; Vol. 87:A008.

Moues CM, Vos MC, van den Bemd GJ, Stijnen T, Hovius SE. Bacterial load in relation to vacuum-assisted closure wound therapy: a prospective randomized trial. Wound Repair and Regeneration 2004;12:11-7.

Moues CM, Vos MC, van den Bemd GJCM, Stijnen T, Hovius SER. Bacterial load in relation to vacuum-assisted closure wound therapy. 13th conference of the European Wound Management Association; 2003 May 22-24; Pisa, Italy. 2003:Presentation 42.

* Moues CM, van den Bemd GCM, Meerding WJ, Hovius SER. An economic evaluation of the use of topical negative pressure on full-thickness wounds. Journal of Wound Care 2005;14:224-7.

Moues CM, van den Bemd GJCM, Heule F, Hovius SER. Comparing conventional gauze therapy to vacuum-assisted closure wound therapy: a prospective randomised trial. Journal of Plastic and Reconstructive Aesthetic Surgery 2007;60:672-81.

Perez 2010 \{published data only\}

Perez D, Bramkamp M, Exe C, von Ruden C, Ziegler A. Modern wound care for the poor: a randomized clinical trial comparing the vacuum system with conventional saline-soaked gauze dressings. American Journal of Surgery 2010;199:14-20.

\section{Rahmanian Schwarz 2012 \{published data only\}}

Rahmanian-Schwarz A, Willkomm LM, Gonser P, Hirt B, Schaller HE. A novel option in negative pressure wound therapy (NPWT) for chronic and acute wound care. Burns 2012;38:573-7. 
Tauro 2007 \{published data only\}

Tauro LF, Ravikrishnan J, Satish Rao BS, Divakar Shenoy H, Shetty SR, Menezes LT. A comparative study of the efficacy of topical negative pressure moist dressings and conventional moist dressings in chronic wounds. Indian Journal of Plastic Surgery 2007;40:133-40.

\section{References to studies awaiting assessment}

Marston 2015 \{published data only\}

Marston WA, Armstrong DG, Reyzelman AM, Kirsner RS. A multicenter randomized controlled trial comparing treatment of venous leg ulcers using mechanically versus electrically powered negative pressure wound therapy. Advances in Wound Care 2015;4:75-82.

\section{Pruksapong 2011 \{published data only\}}

Pruksapong C. Efficacy of portable vacuum dressing in chronic wound care: a prospective randomized control trial. Journal of the Medical Association of Thailand 2011;94:1212-7.

\section{Additional references}

\section{Ashby 2014}

Ashby RL, Gabe R, Ali S, Adderley U, Bland JM, Cullum NA, et al. Clinical and cost-effectiveness of compression hosiery versus compression bandages in treatment of venous leg ulcers (Venous leg Ulcer Study IV, VenUS IV): a randomised controlled trial. The Lancet 2014;383(9920):871-9.

\section{Callam 1987}

Callam MJ, Harper DR, Dale JJ, Ruckley CV. Chronic ulcer of the leg: clinical history. British Medical Journal 1987;294(6584):1389-91.

\section{Coleridge 1988}

Coleridge Smith PD, Thomas P, Scurr JH, Dormandy JA. Causes of venous ulceration: a new hypothesis. $B M J$ 1988;296(6638):1726-7.

\section{Cullum 2014}

Cullum N. Wounds Research for Patient Benefit Programme. Personal communication 2014.

\section{Deeks 2011}

Deeks JJ, Higgins JPT, Altman DG (editors). Chapter 9: Analysing data and undertaking meta-analyses. In: Higgins JPT, Green $\mathrm{S}$ (editors). Cochrane Handbook for Systematic Reviews of Interventions. Version 5.1.0 (updated March 2011). The Cochrane Collaboration, 2011. Available from www.cochranehandbook.org.

\section{Department of Health and Human Services 2009}

Department of Health and Human Services, Office of Inspector General. Comparison of prices for negative pressure wound therapy pumps. https://oig.hhs.gov/oei/reports/ oei-02-07-00660.pdf. OEI-02-07-00660 2009 (accessed Dec 2012).

\section{Dumville 2014}

Dumville JC, Webster J, Evans D, Land L. Negative pressure wound therapy for treating pressure ulcers. Cochrane Database of Systematic Reviews 2014, Issue 10. [DOI: 10.1002/14651858.CD011334]

\section{FDA 2011}

US Food, Drug Administration. FDA Safety Communication: update on serious complications associated with negative pressure wound therapy systems. http://www.fda.gov/ MedicalDevices/Safety/AlertsandNotices/ucm244211.htm 2011 (accessed Nov 2012).

\section{Ghauri 2010}

Ghauri ASK, Nyamekye IK. Leg ulceration: the importance of treating the underlying pathophysiology. Phlebology/Venous Forum of the Royal Society of Medicine 2010;25 Suppl 1:42-51.

\section{Gohel 2007}

Gohel MS, Barwell JR, Taylor M, Chant T, Foy C, Earnshaw JJ, et al. Long term results of compression therapy alone versus compression plus surgery in chronic venous ulceration (ESCHAR): randomised controlled trial. $B M J$ 2007;335(7610):83-7.

\section{Guy 2012}

Guy H. Pressure ulcer risk assessment. Nursing Times 2012;108(4):16-20.

\section{Hahn 2005}

Hahn S, Puffer S, Torgerson DJ, Watson J. Methodological bias in cluster randomised trials. BMC Medical Research Methodology 2005;5:10.

\section{Herber 2007}

Herber OR, Schnepp W, Rieger MA. A systematic review on the impact of leg ulceration on patients' quality of life. Health and Quality of Life Outcomes 2007;5:44.

\section{Higgins 2003}

Higgins JPT, Thompson SG, Deeks JJ, Altman DG. Measuring inconsistency in meta-analyses. BMJ 2003;327(7414):557-60.

\section{Higgins 2011a}

Higgins JPT, Altman DG, Sterne, JAC (editors). Chapter 8: Assessing risk of bias in included studies. In: Higgins JPT, Green S (editors). Cochrane Handbook for Systematic Reviews of Interventions. Version 5.1.0 [updated March 2011]. The Cochrane Collaboration, 2011. Available from www.cochranehandbook.org.

\section{Higgins 2011b}

Higgins JPT, Deeks JJ, Altman DG (editors). Chapter 16: Special topics in statistics. In: Higgins JPT, Green S (editors). Cochrane Handbook for Systematic Reviews of Interventions. Version 5.1.0 [updated March 2011]. The Cochrane Collaboration, 2011. Available from www.cochrane-handbook.org. 


\section{Huang 2014}

Huang C, Leavitt T, Bayer LR, Orgill DP. Effect of negative pressure wound therapy on wound healing. Current Problems in Surgery 2014;51:301-31.

\section{Johnson 1995}

Johnson M. The prevalence of leg ulcers in older people: implications for community nursing. Public Health Nursing 1995;12(4):269-75.

\section{Jull 2012}

Jull AB, Arroll B, Parag V, Waters J. Pentoxifylline for treating venous leg ulcers. Cochrane Database of Systematic Reviews 2012, Issue 12. [DOI: 10.1002/14651858.CD001733.pub3]

\section{KCI 2012}

$\mathrm{KCl}$. Science behind wound therapy. http://www.kcil.com/KCl1/ sciencebehindwoundtherapy (accessed 11 May 2012).

\section{Kontopantelis 2012}

Kontopantelis E, Reeves D. Performance of statistical methods for meta-analysis when true study effects are non-normally distributed: a simulation study. Statistical Methods in Medical Research 2012;21(4):409-26.

\section{Kontopantelis 2013}

Kontopantelis E, Springate DA, Reeves D. A re-analysis of the Cochrane library data: the dangers of unobserved heterogeneity in meta-analysis. PLoS One 2013;8:e69930.

\section{Lefebvre 2011}

Lefebvre C, Manheimer E, Glanville J. Chapter 6: Searching for studies. In: Higgins JPT, Green S (editors). Cochrane Handbook for Systematic Reviews of Interventions. Version 5.1.0 [updated March 2011]. The Cochrane Collaboration, 2011. Available from www.cochrane-handbook.org.

\section{Liberati 2009}

Liberati A, Altman DG, Tetzlaff J, Mulrow C, Gotzsche PC, loannidis JP, et al. The PRISMA statement for reporting systematic reviews and meta-analyses of studies that evaluate health care interventions: explanation and elaboration. PLOS Medicine 2009;6:e1000100.

\section{Madden 2014}

Madden M. Wounds Research for Patient Benefit Programme. Personal Communication 2014.

\section{Margolis 2004}

Margolis DJ, Allen-Taylor L, Hoffstad O, Berlin JA. The accuracy of venous leg ulcer prognostic models in a wound care system. Wound Repair and Regeneration 2004;12(2):163-8.

\section{Moffatt 2004}

Moffatt CJ, Franks PJ, Doherty DC, Martin R, Blewett R, Ross F. Prevalence of leg ulceration in a London population. QJM Monthly Journal of the Association of Physicians 2004;97(7):431.

\section{O'Meara 2012}

O'Meara S, Cullum N, Nelson EA, Dumville JC. Compression for venous leg ulcers. Cochrane Database of Systematic Reviews 2012, Issue 11. [DOI: 10.1002/14651858.CD000265.pub3]

\section{Parmar 1998}

Parmar MK, Torri V, Stewart L. Extracting summary statistics to perform meta-analysis of the published literature for survival endpoints. Statistics in Medicine 1998;17:2815-34.

\section{Peinemann 2008}

Peinemann F, McGauran N, Sauerland S, Lange S. Negative pressure wound therapy: potential publication bias caused by lack of access to unpublished study results data. BMC Medical Research Methodology 2008;8:4.

\section{Persoon 2004}

Persoon A, Heinen MM, van der Vleuten CJM, de Rooij MJ, van de Kerkhof PCM, van Achterberg T. Leg ulcers: a review of their impact on daily life. Journal of Clinical Nursing 2004;13:341-54.

\section{RCN 2006}

The nursing management of patients with venous leg ulcers: recommendations. http://www.rcn.org.uk/__data/assets/pdf_ file/0003/107940/003020.pdf 2006, issue Accessed May 2015.

\section{RevMan 2014 [Computer program]}

The Nordic Cochrane Centre, The Cochrane Collaboration. Review Manager (RevMan) 5.3. Version 5.3. Copenhagen: The Nordic Cochrane Centre, The Cochrane Collaboration, 2014.

\section{Schunemann 2011a}

Schünemann HJ, Oxman AD, Higgins JPT, Vist GE, Glasziou P, Guyatt GH. Chapter 11: Presenting results and 'Summary of findings' tables. In: Higgins JPT, Green S (editors). Cochrane Handbook for Systematic Reviews of Interventions. Version 5.1.0 [updated March 2011]. The Cochrane Collaboration, 2011. Available from www.cochrane-handbook.org.

\section{Schunemann 2011b}

Schünemann HJ, Oxman AD, Higgins JPT, Deeks JJ, Glasziou P, Guyatt GH. Chapter 12: Interpreting results and drawing conclusions. In: Higgins JPT, Green S (editors). Cochrane Handbook for Systematic Reviews of Interventions. Version 5.1.0 [updated March 2011]. The Cochrane Collaboration, 2011. Available from www.cochrane-handbook.org.

\section{SIGN 2010}

Management of chronic venous leg ulcers: a national clinical guideline. http://www.sign.ac.uk/pdf/sign120.pdf 2010, issue Accessed May 2015.

\section{SIGN 2012}

Scottish Intercollegiate Guidelines Network (SIGN). Search filters. http://www.sign.ac.uk/methodology/ filters.html\#random (accessed June 2014).

\section{Srinivasaiah 2007}

Srinivasaiah N, Dugdall H, Barrett S, Drew PJ. A point prevalence survey of wounds in north-east England. Journal of Wound Care 2007;16(10):413-6, 418-9. 


\section{Sterne 2011}

Sterne JAC, Egger M, Moher D. Chapter 10: Addressing reporting biases. In: Higgins JPT, Green S (editors). Cochrane Handbook for Systematic Reviews of Interventions. Version 5.1.0 [updated March 2011]. The Cochrane Collaboration, 2011. Available from www.cochrane-handbook.org.

\section{Thompson 1999}

Thompson SG, Sharp SJ. Explaining heterogeneity in metaanalysis: a comparison of methods. Statistics in Medicine 1999;18:2693-708.

\section{Ubbink 2008}

Ubbink DT, Westerbos SJ, Evans D, Land L, Vermeulen H. Topical negative pressure for treating chronic wounds. Cochrane Database of Systematic Reviews 2008, Issue 3. [DOI: 10.1002/14651858.CD001898.pub2]

\section{CHARACTERISTICS OF STUDIES}

Characteristics of included studies [ordered by study ID]

\section{Vowden 2009}

Vowden KR, Vowden P. The prevalence, management and outcome for patients with lower limb ulceration identified in a wound care survey within one English health care district. Journal of Tissue Viability 2009;18(1):13-9.

\section{Walker 2002}

Walker N, Rodgers A, Birchall N, Norton R, MacMahon S. The occurrence of leg ulcers in Auckland: results of a population-based study. New Zealand Medical Journal 2002;115(1151):159-62.

\section{Woo 2013}

Woo KY, Alavi A, Evans R, Despatis M, Allen J. New advances in compression therapy for venous leg ulcers. Surgical Technology International 2007;335(7610):61-8.

* Indicates the major publication for the study

Vuerstaek 2006

Methods
Multi-centred, conducted in hospital settings in one geographical location in Netherlands
Duration of follow-up was a maximum of 12 months

Participants

60 participants: the study report lists 54 included in analysis for complete wound healing; 47 analyzed at 12-month follow-up

\section{Inclusion criteria:}

- patients hospitalised with chronic venous, combined venous and arterial, or microangiopathic (arteriolosclerotic) leg ulcers of $>6$ months' duration after surgical treatment options had been exhausted and extensive ambulatory treatment (> 6 months) in an outpatient clinic (according to the Scottish Intercollegiate Guideline Network (SIGN)) had failed

- patients with venous or combined venous/arterial leg ulcers were treated with multilayer, short, stretch bandages. If the ulcer did not reduce in size after 6 months of ambulatory treatment, patients were hospitalised to add bed rest and skin-grafting to their treatment and became eligible for entry in the study

- in patients presenting with multiple ulcerations, the clinically most severe CLU was included in the study

\section{Exclusion criteria:}

- ulcer chronicity $<6$ months duration

- age $>85$ years old

- use of immune suppression

- allergy to wound products

\section{Baseline details}

Sex: $23 \%$ participants were male (both trial arms)

Median age (minimum-maximum): NPWT group: 74 years (53-81); standard care group: 72 years (45-83)

Diabetes: $5 \%$ of participants had diabetes (both arms) 
Vuerstaek 2006 (Continued)

Median ulcer chronicity at inclusion (months; minimum-maximum): NPWT group: 8 (6-24); standard care group: 7 (6-12)

Median ulcer surface (length width $\mathrm{cm}^{2}$; minimum-maximum): NPWT group: 33 (2-150); standard care group: 43 (3-250)

Interventions

Group A: VAC Therapy ( $\mathrm{KCI}$ Inc TX, USA; $n=30$ ). Perminant negative pressure of $125 \mathrm{mmHg}$ until the wound was $100 \%$ granulated. A punch skin-graft transplant then took place and was covered with a non-adhesive dressing. The study then reports that after 4 days of continuous subatmospheric pressure, once all skin grafts were attached well, standard wound care was continued using a non-adhesive dressing (Atrauman; Hartmann, Nijmegen, The Netherlands) and a multiple-layer compression bandage (Rosidal K; Lohmann \& Rauscher, Rengsdorf, Germany)

Group B: standard care $(n=30)$. Daily local wound care according to the SIGN guideline, and compression therapy were applied until the wound was $100 \%$ granulated. Once $100 \%$ granulation was achieved and minimal wound secretion was seen, these participants also received punch skin-graft transplant covered with a non-adhesive dressing (Atrauman) and compression therapy. The inner dressing was not changed for 4 days. Once all skin grafts had attached well, standard wound care was continued using a non-adhesive dressing (Atrauman) and a multilayer compression bandage (Rosidal K), when possible, until complete healing

Primary outcomes
- Complete wound healing (time to healing in days with healing defined as $100 \%$ epithelialisation)
- Adverse events (defined as complications, but not clear if reported at the participant level)

\section{Secondary outcomes}

- Health-related quality of life - utility measured using EQ-5D

- Mean costs

- Mean pain scores

- Readiness for surgery (defined as time taken to prepare wound for skin graft)

Notes Only 1 ulcer per participant was followed

Funding source: the study was supported by the Dutch department of Kinetic Concepts, Inc (KCl)

\section{Risk of bias}

\begin{tabular}{lll}
\hline Bias & Authors' judgement & Support for judgement \\
\hline $\begin{array}{l}\text { Random sequence genera- } \\
\text { tion (selection bias) }\end{array}$ & Low risk & $\begin{array}{l}\text { Quote: "patients were randomly assigned to the V.A.C. group or to the control } \\
\text { group (standard wound care) by a computer program using random permut- } \\
\text { ed blocks of eight. Randomization was carried out within three strata, one for } \\
\text { each ulcer type: venous, combined venous/arterial, and arteriolosclerotic ul- } \\
\text { cers." }\end{array}$ \\
$\begin{array}{l}\text { Comment: a random component using a computer program in the sequence } \\
\text { generation process described }\end{array}$ \\
$\begin{array}{l}\text { Allocation concealment } \\
\text { (selection bias) }\end{array}$ & Low risk & $\begin{array}{l}\text { Quote: "Treatment allocation occurred through telephone calls to the coordi- } \\
\text { nating center." }\end{array}$ \\
& Comment: central allocation was used to conceal allocation
\end{tabular}

\begin{tabular}{|c|c|c|}
\hline Blinding of outcome as- & Unclear risk & Complete wound healing \\
\hline All outcomes & & $\begin{array}{l}\text { Quote: "Because masking the interventions was not possible, patients were } \\
\text { reviewed clinically by the same independent research physician and consul- } \\
\text { tant dermatologist twice a week until wound closure. Thereafter, the same re- } \\
\text { search physician prospectively monitored the patients at } 3,6 \text {, and } 12 \text { months }\end{array}$ \\
\hline
\end{tabular}


after discharge. All participating clinicians completed standardized case record forms during their control visits, treatments, and follow-ups.'

Comment: key study personnel were not blinded but the outcome assessment seems to have been blinded. It is not clear whether this extended to decisions made regarding time to wound bed preparation, and thus skin grafting, but the report suggests so. The author has been contacted for clarification.

Incomplete outcome data Unclear risk (attrition bias)

All outcomes
Comment: 6 participants were lost to follow-up ( 2 from one group and 4 from another); time to event methods outlined suggest an ITT analysis was undertaken as far as was possible. The impact of these missing data on the outcomes is not clear. Only 47 participants were reported as analyzed at 12 months.

\begin{tabular}{lll}
\hline $\begin{array}{l}\text { Selective reporting (re- } \\
\text { porting bias) }\end{array}$ & Low risk & $\begin{array}{l}\text { Comment: protocol not obtained, based on trial report only, but trial report in- } \\
\text { cluded all expected outcomes }\end{array}$ \\
\hline Other bias & Low risk & No unit of analyses issues \\
\hline
\end{tabular}

\section{Abbreviations}

EQ-5D: Euro-qol

Characteristics of excluded studies [ordered by study ID]

\begin{tabular}{ll}
\hline Study & Reason for exclusion \\
\hline Armstrong 2012 & Included multiple wounds types. Unable to obtain leg ulcer data separately \\
\hline Braakenburg 2006 & Included multiple wounds types. Unable to obtain leg wound data separately \\
\hline de Laat 2011 & Wound type was not relevant \\
\hline Dini 2009 & $\begin{array}{l}\text { No relevant outcomes reported: conference abstract reported healing as an outcome, but data not } \\
\text { available }\end{array}$ \\
\hline Hu 2009 & Study population had range of wounds - not able to isolate leg ulcer data - based on translation \\
\hline Joseph 2000 & Included multiple wounds types. Unable to obtain leg ulcer wound data separately \\
\hline Lantis 2004 & No relevant outcomes \\
\hline Moues 2005 & Included multiple wounds types. Unable to obtain leg ulcer wound data separately \\
\hline Perez 2010 & Included multiple wounds types. Unable to obtain leg ulcer wound data separately \\
\hline Rahmanian Schwarz 2012 & Included multiple wounds types. Unable to obtain leg ulcer wound data separately \\
\hline Tauro 2007 & Not an RCT \\
\hline
\end{tabular}

\section{Abbreviation}

$\mathrm{RCT}$ : randomized controlled trial

Characteristics of studies awaiting assessment [ordered by study ID] 
Marston 2015

\begin{tabular}{ll}
\hline Methods & RCT \\
\hline Participants & $\begin{array}{l}\text { The paper suggests that people with venous leg ulcers were randomized along with people with } \\
\text { foot ulcers. However, the paper only reported } \\
\text { data for those with VLU - which is a sub-group of the overall population. This might impact on the } \\
\text { randomized nature of the study }\end{array}$ \\
\hline Interventions & 2 types of NPWT \\
\hline Outcomes & Time to healing \\
\hline Notes & Authors contacted to clarify the design and the number of people with foot ulcers recruited \\
\hline
\end{tabular}

Pruksapong 2011

Methods Unable to locate full study to date

\section{Participants}

Interventions

\section{Outcomes}

Notes

\section{Abbreviations}

NPWT: negative pressure wound therapy

RCT: randomized controlled trial

VLU: venous leg ulcer

\section{DATA AND ANALYSES}

\section{Comparison 1. NPWT compared with standard care}

\begin{tabular}{|c|c|c|c|c|}
\hline Outcome or subgroup title & No. of studies & $\begin{array}{l}\text { No. of partici- } \\
\text { pants }\end{array}$ & Statistical method & Effect size \\
\hline $\begin{array}{l}1 \text { Proportion of wounds completely } \\
\text { healed (up to } 12 \text {-month follow-up) }\end{array}$ & 1 & & $\begin{array}{l}\text { Risk Ratio (M-H, Fixed, } \\
95 \% \mathrm{Cl})\end{array}$ & Subtotals only \\
\hline $\begin{array}{l}2 \text { Health related quality of life/health sta- } \\
\text { tus }\end{array}$ & 1 & & $\begin{array}{l}\text { Mean Difference (IV, Fixed, } \\
95 \% \mathrm{CI})\end{array}$ & Subtotals only \\
\hline $\begin{array}{l}3 \text { Pain scores (Present Pain Intensity In- } \\
\text { strument) }\end{array}$ & 1 & & $\begin{array}{l}\text { Mean Difference (IV, Fixed, } \\
95 \% \mathrm{CI})\end{array}$ & Subtotals only \\
\hline
\end{tabular}


Analysis 1.1. Comparison 1 NPWT compared with standard care, Outcome 1 Proportion of wounds completely healed (up to 12-month follow-up).

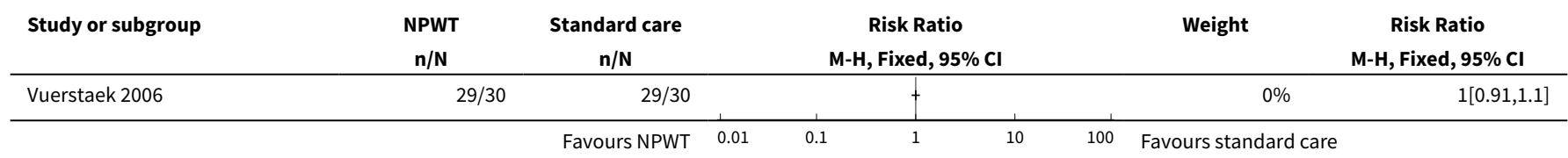

Analysis 1.2. Comparison 1 NPWT compared with standard care, Outcome 2 Health related quality of life/health status.

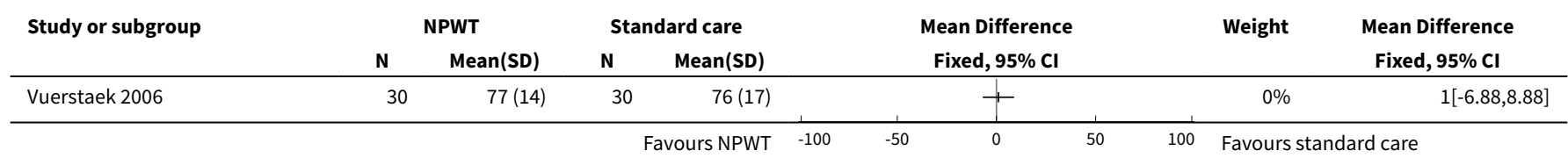

Analysis 1.3. Comparison 1 NPWT compared with standard care, Outcome 3 Pain scores (Present Pain Intensity Instrument).

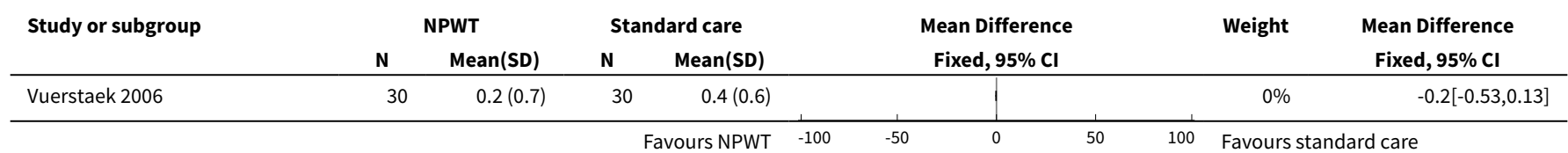




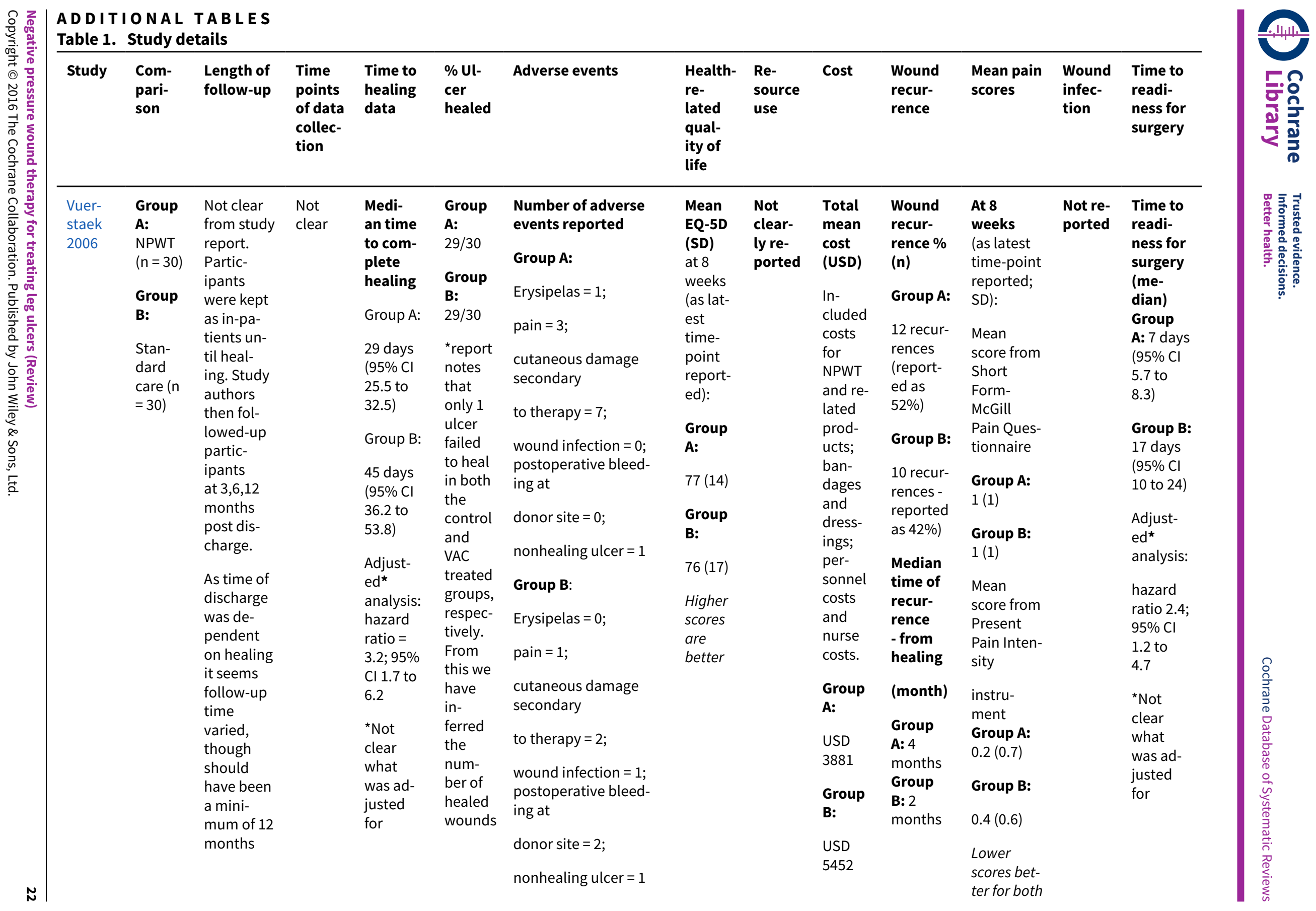




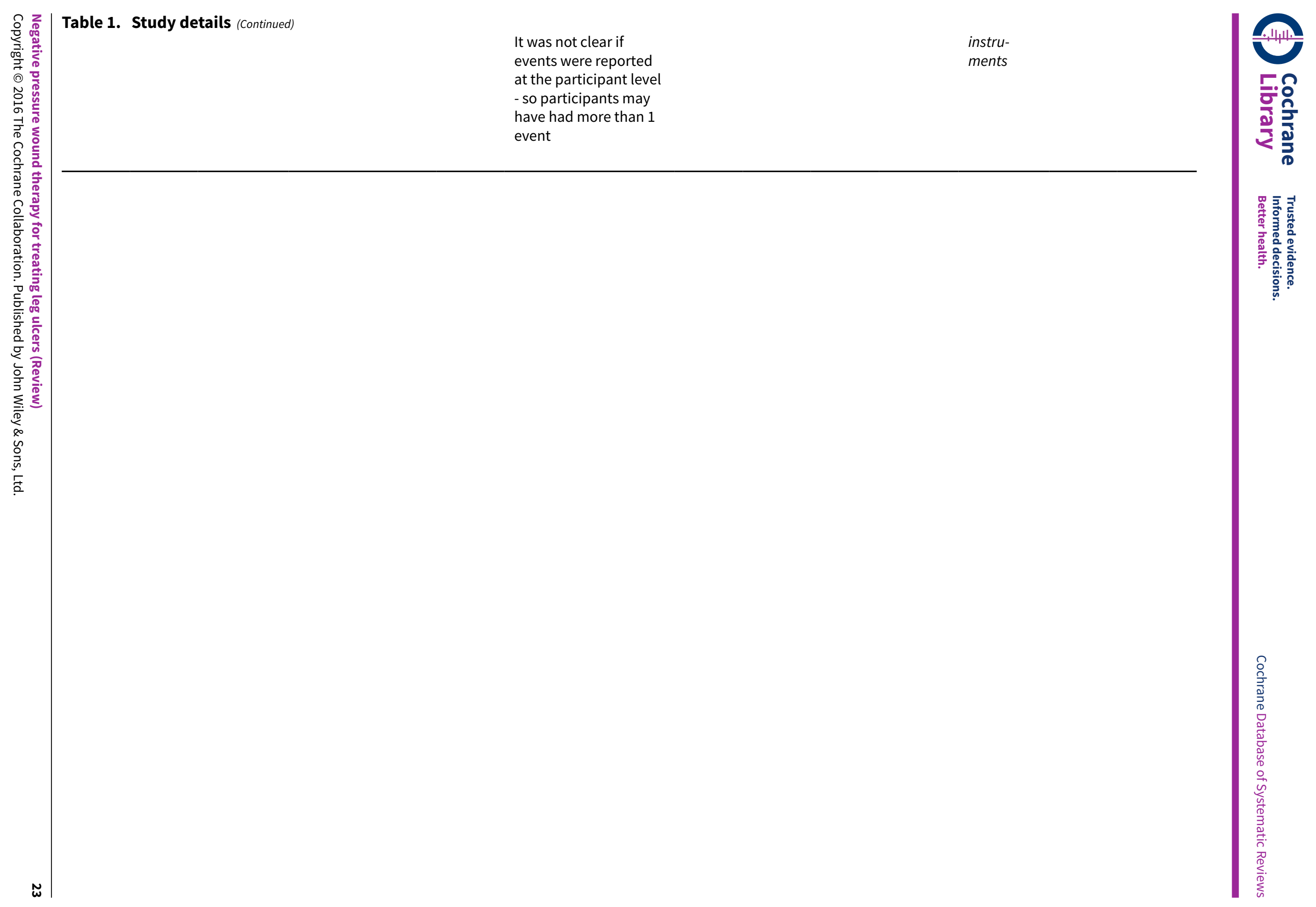




\section{AP PEN DICES}

\section{Appendix 1. Risk of bias assessment (individually randomised controlled trials)}

\section{Was the allocation sequence randomly generated?}

\section{Low risk of bias}

The investigators describe a random component in the sequence generation process such as: referring to a random number table; using a computer random number generator; coin tossing; shuffling cards or envelopes; throwing dice; drawing of lots.

\section{High risk of bias}

The investigators describe a non-random component in the sequence generation process. Usually, the description would involve some systematic, non-random approach, for example: sequence generated by odd or even date of birth; sequence generated by some rule based on date (or day) of admission; sequence generated by some rule based on hospital or clinic record number.

\section{Unclear}

Insufficient information about the sequence generation process available to permit judgement of low or high risk of bias.

\section{Was the treatment allocation adequately concealed?}

\section{Low risk of bias}

Participants and investigators enrolling participants could not foresee assignment because one of the following, or an equivalent method, was used to conceal allocation: central allocation (including telephone, web-based and pharmacy-controlled randomisation); sequentially numbered drug containers of identical appearance; sequentially numbered, opaque, sealed envelopes.

\section{High risk of bias}

Participants or investigators enrolling participants could possibly foresee assignments and thus introduce selection bias, such as allocation based on: using an open random allocation schedule (e.g. a list of random numbers); assignment envelopes without appropriate safeguards (e.g. if envelopes were unsealed or non opaque or not sequentially numbered); alternation or rotation; date of birth; case record number; any other explicitly unconcealed procedure.

\section{Unclear}

Insufficient information available to permit judgement of low or high risk of bias. This is usually the case if the method of concealment is not described or not described in sufficient detail to allow a definite judgement, for example if the use of assignment envelopes is described, but it remains unclear whether envelopes were sequentially numbered, opaque and sealed.

\section{Blinding - was knowledge of the allocated interventions adequately prevented during the study?}

\section{Low risk of bias}

Any one of the following:

- No blinding, but the review authors judge that the outcome and the outcome measurement are not likely to be influenced by lack of blinding.

- Blinding of participants and key study personnel ensured, and unlikely that the blinding could have been broken.

- Either participants or some key study personnel were not blinded, but outcome assessment was blinded and the non-blinding of others unlikely to introduce bias.

\section{High risk of bias}

Any one of the following:

- No blinding or incomplete blinding, and the outcome or outcome measurement is likely to be influenced by lack of blinding.

- Blinding of key study participants and personnel attempted, but likely that the blinding could have been broken.

- Either participants or some key study personnel were not blinded, and the non-blinding of others likely to introduce bias.

\section{Unclear}

Either of the following:

- Insufficient information to permit judgement of low or high risk of bias.

- The study did not address this outcome. 


\section{Were incomplete outcome data adequately addressed?}

\section{Low risk of bias}

Any one of the following:

- No missing outcome data.

- Reasons for missing outcome data unlikely to be related to true outcome (for survival data, censoring unlikely to be introducing bias).

- Missing outcome data balanced in numbers across intervention groups, with similar reasons for missing data across groups.

- For dichotomous outcome data, the proportion of missing outcomes compared with observed event risk not enough to have a clinically relevant impact on the intervention effect estimate.

- For continuous outcome data, plausible effect size (difference in means or standardised difference in means) among missing outcomes is not enough to have a clinically relevant impact on observed effect size.

- Missing data have been imputed using appropriate methods.

\section{High risk of bias}

Any one of the following:

- Reason for missing outcome data likely to be related to true outcome, with either imbalance in numbers or reasons for missing data across intervention groups.

- For dichotomous outcome data, the proportion of missing outcomes compared with observed event risk enough to induce clinically relevant bias in intervention effect estimate.

- For continuous outcome data, plausible effect size (difference in means or standardised difference in means) among missing outcomes enough to induce clinically relevant bias in observed effect size.

- 'As-treated' analysis done with substantial departure of the intervention received from that assigned at randomisation.

- Potentially inappropriate application of simple imputation.

\section{Unclear}

Either of the following:

- Insufficient reporting of attrition/exclusions to permit judgement of low or high risk of bias (e.g. number randomized not stated, no reasons for missing data provided).

- The study did not address this outcome.

\section{Are reports of the study free of suggestion of selective outcome reporting?}

\section{Low risk of bias}

Either of the following:

- The study protocol is available and all of the study's pre-specified (primary and secondary) outcomes that are of interest in the review have been reported in the pre-specified way.

- The study protocol is not available but it is clear that the published reports include all expected outcomes, including those that were pre-specified (convincing text of this nature may be uncommon).

\section{High risk of bias}

Any one of the following:

- Not all of the study's pre-specified primary outcomes have been reported.

- One or more primary outcomes are reported using measurements, analysis methods or subsets of the data (e.g. subscales) that were not pre-specified.

- One or more reported primary outcomes were not pre-specified (unless clear justification for their reporting is provided, such as an unexpected adverse effect).

- One or more outcomes of interest in the review are reported incompletely so that they cannot be entered in a meta-analysis.

- The study report fails to include results for a key outcome that would be expected to have been reported for such a study.

\section{Unclear}

Insufficient information available to permit judgement of low or high risk of bias. It is likely that the majority of studies will fall into this category. 


\section{Other sources of potential bias}

\section{Low risk of bias}

The study appears to be free of other sources of bias.

\section{High risk of bias}

There is at least one important risk of bias. For example, the study:

- had a potential source of bias related to the specific study design used; or

- has been claimed to have been fraudulent; or

- had some other problem.

\section{Unclear}

There may be a risk of bias, but there is either:

- insufficient information to assess whether an important risk of bias exists; or

- insufficient rationale or evidence that an identified problem will introduce bias.

\section{Appendix 2. Risk of bias (cluster randomised controlled trials)}

\section{Types of bias in cluster-randomised trials}

In cluster-randomised trials, particular biases to consider include:

- recruitment bias;

- baseline imbalance;

- loss of clusters;

- incorrect analysis; and

- comparability with individually randomized trials.

\section{Recruitment bias}

Recruitment bias can occur when individuals are recruited to the trial after the clusters have been randomized, as knowledge about whether each cluster is an 'intervention' or 'control' cluster could affect the types of participants recruited.

\section{Baseline imbalance}

Cluster-randomised trials often randomise all clusters at once, so lack of concealment of an allocation sequence should not usually be an issue. However, because small numbers of clusters are randomized, there is a possibility of chance baseline imbalance between the randomized groups, in terms of either the clusters or the individuals. Although this is not a form of bias, as such, the risk of baseline differences can be reduced by using stratified or pair-matched randomisation of clusters. Reporting of the baseline comparability of clusters, or statistical adjustment for baseline characteristics, can help reduce concern about the effects of baseline imbalance.

\section{Loss of clusters}

Occasionally complete clusters are lost from a trial, and have to be omitted from the analysis. Just as for missing outcome data in individually-randomised trials, this may lead to bias. In addition, missing outcomes for individuals within clusters may also lead to a risk of bias in cluster-randomised trials.

\section{Incorrect analysis}

Many cluster-randomised trials are analyzed by incorrect statistical methods, that do not take the clustering into account. Such analyses create a 'unit of analysis error' and produce over-precise results (the standard error of the estimated intervention effect is too small) and P values that are too small. They do not lead to biased estimates of effect, but if they remain uncorrected, they will receive too much weight in a meta-analysis.

\section{Comparability with individually randomized trials}

In a meta-analysis including both cluster- and individually-randomised trials, or including cluster-randomised trials with different types of clusters, possible differences between the intervention effects being estimated need to be considered. For example, in a vaccine trial of infectious diseases, a vaccine applied to all individuals in a community would be expected to be more effective than if the vaccine was applied to only half of the people. Another example is provided by a Cochrane review of hip protectors (Hahn 2005). The cluster trials showed a large positive effect, whereas individually-randomised trials did not show any clear benefit. One possibility is that there was a 'herd effect' in the cluster-randomised trials (which were often performed in nursing homes, where compliance with using the protectors may have been enhanced). In general, such 'contamination' would lead to underestimates of effect. Thus, if an intervention effect is still 
demonstrated despite contamination in those trials that were not cluster-randomised, a confident conclusion about the presence of an effect can be drawn. However, the size of the effect is likely to be underestimated. Contamination and 'herd effects' may be different for different types of cluster.

\section{Appendix 3. Search strategies}

Database: Ovid MEDLINE(R) <1946 to July Week 2 2014>

1 exp Negative-Pressure Wound Therapy/

2 exp Suction/

3 exp Vacuum/

4 (negative pressure or negative-pressure or TNP or NPWT).tw.

5 (sub-atmospheric or subatmospheric).tw.

$6\left(\left(\right.\right.$ seal $^{\star}$ adj surface $\left.{ }^{\star}\right)$ or $\left(\right.$ seal $^{\star}$ adj aspirat $\left.\left.{ }^{\star}\right)\right) . t w$.

7 (wound adj2 suction*).tw.

8 (wound adj5 drainage).tw.

9 ((foam adj suction) or (suction adj dressing $\left.\left.{ }^{\star}\right)\right)$. tw.

10 (vacuum assisted closure technique or VAC).tw.

11 ((vacuum adj therapy) or (vacuum adj dressing*) or (vacuum adj seal ${ }^{\star}$ ) or (vacuum adj closure) or (vacuum adj compression) or (vacuum adj pack ${ }^{\star}$ ) or (vacuum adj drainage) or (suction* adj drainage)).tw.

12 or/1-11

13 exp Leg Ulcer/

14 (varicose ulcer* ${ }^{\star}$ r venous ulcer ${ }^{\star}$ or leg ulcer ${ }^{\star}$ or stasis ulcer ${ }^{\star}$ or crural ulcer ${ }^{\star}$ or ulcus cruris or ulcer cruris).tw.

1513 or 14

1612 and 15

17 randomized controlled trial.pt.

18 controlled clinical trial.pt.

19 randomi?ed.ab.

20 placebo.ab.

21 clinical trials as topic.sh.

22 randomly.ab.

23 trial.ti.

24 exp animals/ not humans.sh.

2516 and 24

Database: Embase <1974 to 2014 July 21>

1 exp Suction drainage/

2 exp Vacuum assisted closure/ (

3 (negative pressure or negative-pressure or TNP or NPWT).tw.

4 (sub-atmospheric or subatmospheric).tw.

5 ((seal\$ adj surface\$) or (seal\$ adj aspirat\$)).tw.

6 (wound adj2 suction\$).tw.

7 (wound adj5 drainage).tw.

8 ((foam adj suction) or (suction adj dressing\$)).tw.

9 (vacuum assisted closure technique or VAC).tw.

10 ((vacuum adj therapy) or (vacuum adj dressing\$) or (vacuum adj seal\$) or (vacuum adj closure) or (vacuum adj compression) or (vacuum adj pack\$) or (vacuum adj drainage) or (suction\$ adj drainage)).tw.

11 or/1-10

12 exp Leg Ulcer/

13 (varicose ulcer $^{\star}$ or venous ulcer ${ }^{\star}$ or leg ulcer ${ }^{\star}$ or stasis ulcer ${ }^{\star}$ or crural ulcer $^{\star}$ or ulcus cruris or ulcer cruris).tw.

1412 or 13

1511 and 14

16 Randomized controlled trials/

17 Single-Blind Method/

18 Double-Blind Method/

19 Crossover Procedure/

20 (random\$ or factorial\$ or crossover\$ or cross over\$ or cross-over\$ or plac\$ or assign\$ or allocat\$ or volunteer\$).ti,ab.

21 (doubl\$ adj blind\$).ti,ab.

22 (singl\$ adj blind\$).ti,ab.

23 or/16-22

24 exp animals/ or exp invertebrate/ or animal experiment/ or animal model/ or animal tissue/ or animal cell/ or nonhuman/

25 human/ or human cell/

26 and/24-25

Negative pressure wound therapy for treating leg ulcers (Review) 
CINAHL: July 22, 2014

S29 S16 AND S28

$\mathrm{S} 28 \mathrm{~S} 17$ or $\mathrm{S} 18$ or $\mathrm{S} 19$ or $\mathrm{S} 20$ or S21 or S22 or S23 or S24 or S25 or S26 or S27

S27 MH "Quantitative Studies"

S26 TI placebo* or AB placebo*

S25 MH "Placebos"

S24 TI random ${ }^{\star}$ allocat* or AB random ${ }^{\star}$ allocat ${ }^{\star}$

S23 MH "Random Assignment"

S22 $\mathrm{TI}$ randomi?ed control ${ }^{\star}$ trial $^{*}$ or $\mathrm{AB}$ randomi?ed control ${ }^{\star}$ trial $^{*}$

S21 AB ( singl* or doubl* or trebl* or tripl ${ }^{*}$ ) and $A B$ ( blind ${ }^{*}$ or mask* )

S20 TI ( singl $l^{*}$ or doubl* or trebl ${ }^{\star}$ or tripl ${ }^{\star}$ ) and TI ( blind ${ }^{\star}$ or mask ${ }^{\star}$ )

S19 TI clinic ${ }^{\star} \mathrm{N} 1$ trial $^{\star}$ or AB clinic ${ }^{\star} \mathrm{N} 1$ trial $^{\star}$

S18 PT Clinical trial

S17 MH "Clinical Trials+"

S16 S12 and S15

$\mathrm{S} 15 \mathrm{~S} 13$ or $\mathrm{S} 14$

S14 TI (varicose ulcer ${ }^{\star}$ or venous ulcer* or leg ulcer ${ }^{\star}$ or stasis ulcer ${ }^{\star}$ or crural ulcer ${ }^{\star}$ or ulcer cruris or ulcus cruris) or AB (varicose ulcer ${ }^{\star}$ or venous ulcer ${ }^{\star}$ or leg ulcer ${ }^{\star}$ or stasis ulcer ${ }^{\star}$ or crural ulcer* or ulcer cruris or ulcus cruris)

S13 (MH "Leg Ulcer+")

$\mathrm{S} 12 \mathrm{~S} 1$ or $\mathrm{S} 2$ or $\mathrm{S} 3$ or $\mathrm{S} 4$ or $\mathrm{S} 5$ or $\mathrm{S} 6$ or $\mathrm{S} 7$ or $\mathrm{S} 8$ or $\mathrm{S} 9$ or $\mathrm{S} 10$ or $\mathrm{S} 11$

$\mathrm{S} 11 \mathrm{TI}$ foam suction or suction dressing* or suction drainage or AB foam suction or suction dressing* or suction drainage

S10 AB vacuum therapy or vacuum dressing* or vacuum seal* or vacuum closure or vacuum compression or vacuum pack or vacuum drainage

S9 TI vacuum therapy or vacuum dressing ${ }^{*}$ or vacuum seal* or vacuum closure or vacuum compression or vacuum pack or vacuum drainage S8 TI wound N5 drainage or AB wound N5 drainage

S7 TI wound N5 suction* or AB wound N5 suction*

S6 TI seal ${ }^{*} \mathrm{~N} 1$ surface $^{\star}$ or seal ${ }^{*} \mathrm{~N} 1$ aspirat $^{\star}$ or AB seal ${ }^{*} \mathrm{~N} 1$ surface$^{*}$ or seal ${ }^{*} \mathrm{~N} 1$ aspirat $^{\star}$

S5 TI sub-atmospheric or subatmospheric or AB sub-atmospheric or subatmospheric

S4 TI negative pressure or negative-pressure or TNP or AB negative pressure or negative-pressure or TNP

S3 (MH "Negative Pressure Wound Therapy")

$\mathrm{S} 2$ (MH "Vacuum")

S1 (MH "Suction+")

\section{CONTRIBUTIONSOF AUTHORS}

Jo Dumville: conceived the review question; developed and co-ordinated the protocol; secured funding; completed the first draft of the protocol; is the guarantor of the protocol; and approved the final version of the protocol prior to submission.

Lucy Land: conceived the review question and developed the protocol; performed part of writing and editing the protocol; approved the final version of the protocol prior to submission.

Debra Evans: conceived the review question and developed the protocol; performed part of writing and editing the protocol; approved the final version of the protocol prior to submission.

Frank Peinemann: conceived the review question and developed the protocol; completed the first draft of the protocol; approved the final version of the protocol prior to submission.

\section{Contributions of editorial base}

Joan Webster, Editor: advised on methodology, interpretation and protocol content; and approved the final protocol prior to submission. Sally Bell-Syer: co-ordinated the editorial process; advised on methodology, interpretation and content; and edited the protocol. Amanda Briant: designed the search strategy.

\section{DECLARATIONS OF INTEREST}

Jo Dumville is funded as part of the NIHR Cochrane Programme Grant Project: 13/89/08 - High Priority Cochrane Reviews in Wound Prevention and Treatment.

Lucy Land: nothing to declare.

Debra Evans: nothing to declare.

Frank Peinemann: nothing to declare. 


\section{SOURCES OF SUPPORT}

\section{Internal sources}

- School of Nursing, Midwifery and Social Work, University of Manchester, UK.

\section{External sources}

- This project was supported by the National Institute for Health Research, via Cochrane Infrastructure and Cochrane Programme Grant funding (NIHR Cochrane Programme Grant 13/89/08 - High Priority Cochrane Reviews in Wound Prevention and Treatment) to Cochrane Wounds. The views and opinions expressed therein are those of the authors and do not necessarily reflect those of the Systematic Reviews Programme, NIHR, NHS or the Department of Health., UK.

- The National Insitiute for Health Research (NIHR) is the sole funder of the Cochrane Wounds Group, UK.

\section{DIFFERENCES BETWEEN PROTOCOL AND REVIEW}

We added some outcomes to this review based on changes we made to another review pertaining to how we treat adverse event data. We also added an outcome on time to healing/preparation for surgery, as there is increasing recognition that this is a clinically important outcome; we have also added this outcome to other NPWT reviews.

The following sentence was removed from the protocol (type of participant section) while we consider whether undertaking this would resulting in including essentially non-randomised data:

"Studies recruiting participants with leg ulcers alongside people with other types of wounds were included if the data for people with leg ulcers were presented separately (or available from the study authors)."

One study with two arms (individually randomised) was included in this review - thus planned data pooling and investigation of reporting biases, heterogeneity and sub-group analysis that were planned where possible were not conducted.

\section{NOTES}

This review replaces the earlier review: Topical negative pressure for treating chronic wounds (Ubbink 2008).

\section{INDEX TERMS}

\section{Medical Subject Headings (MeSH)}

${ }^{\star}$ Negative-Pressure Wound Therapy; Leg Ulcer ["therapy]; Randomized Controlled Trials as Topic

\section{MeSH check words}

Humans 